\title{
Merged neutral beams
}

\author{
Andreas Osterwalder
}

\section{Correspondence:}

andreas.osterwalder@epfl.ch

Institute for Chemical Sciences and Engineering, Ecole Polytechnique

Fédérale de Lausanne (EPFL), 1015

Lausanne, Switzerland

\section{照 Springer}

\begin{abstract}
A detailed description of a merged beam apparatus for the study of low energy molecular scattering is given. This review is intended to guide any scientist who plans to construct a similar experiment, and to provide some inspiration in describing the approach we chose to our goal. In our experiment a supersonic expansion of paramagnetic particles is merged with one of polar molecules. A magnetic and an electric multipole guide are used to bend the two beams onto the same axis. We here describe in detail how the apparatus is designed, characterised, and operated.
\end{abstract}

Keywords: Cold molecules; Low energy scattering; Molecular beams; Cold chemistry

\section{Review}

In the last 15 years several methods have been developed to prepare molecular samples at temperatures well below $1 \mathrm{~K}$ and to completely control their translational degrees of freedom [1-4]. A particular area where this is of interest is the study of molecular collisions, and cold chemistry [4-11]. Ultracold molecules, produced by joining two atoms at very low temperature, enabled the investigation of molecular collisions at temperatures as low as $1 \mu \mathrm{K}$ [12-14]. These methods are ideally suited to study alkali dimers, but in order to access broader classes of molecules and a larger range of temperatures a different approach was necessary. The present paper describes such an approach, namely the merging of two molecular beams with controlled velocity.

One of the most important developments for reaction dynamics studies in the twentieth century was the crossed-beam technique, for which a Nobel prize was awarded in 1986. In this experiment two molecular beams [15] are crossed to enable a highly detailed investigation of gas-phase molecular scattering, and in particular the measurement of state-to-state differential cross sections [16, 17]. The high velocities of supersonic expansions and the crossing angle of usually 90 degrees make the crossed beam technique ideal for studies at high collision energies $\left(E / k_{B} \gtrsim 100 \mathrm{~K}\right.$, where $k_{B}$ is the Boltzmann constant). Lower energies are not accessible without special modifications, thus making the method blind to some of the most fundamental quantum mechanical effects in molecular scattering.

The collision energy in a crossed beam experiment depends on the relative velocity of the two reactants. In any molecular collision event the energetics are given only by the motion in the molecular frame of reference (MFR), but not that of the centre of mass (CM). This is illustrated in Fig. 1. Panel A shows the position vectors for reactants 1 and 2

(c) 2015 Osterwalder. licensee Springer on behalf of EPJ. This is an Open Access article distributed under the terms of the Creative Commons Attribution License (http://creativecommons.org/licenses/by/4.0), which permits unrestricted use, distribution, and reproduction in any medium, provided the original work is properly credited. 
A

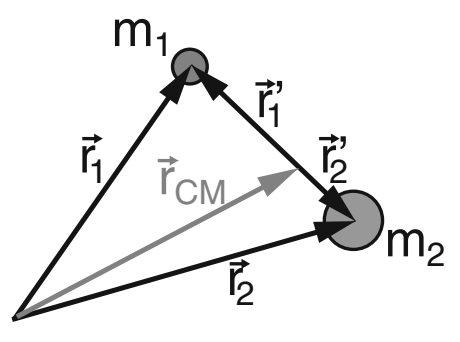

B

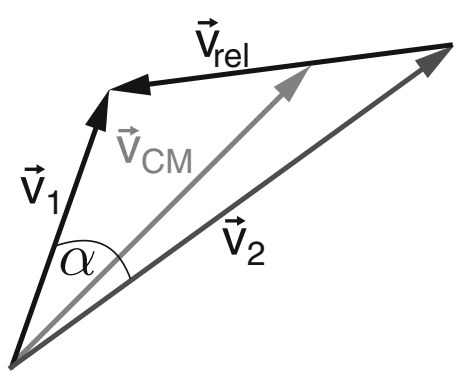

Fig. 1 Position and velocity diagrams for a molecular collision. a position vectors for two colliding particles in the lab frame and in the center-of-mass frame. $\mathbf{b}$ velocities for the same two particles in the lab frame, and the relative velocity vector $\vec{v}$

in the laboratory frame of reference (LFR) as $\vec{r}_{1}$ and $\vec{r}_{2}$, and in the CM frame of reference as $\vec{r}_{1}^{\prime}$ and $\vec{r}_{2}^{\prime}$. The collision energy is given as

$$
E_{\text {coll }}=\frac{\mu}{2}\left|\vec{v}_{\text {rel }}\right|^{2}=\frac{\mu}{2}\left(\left|\vec{v}_{1}\right|^{2}+\left|\vec{v}_{2}\right|^{2}-2\left|\vec{v}_{1}\right|\left|\vec{v}_{2}\right| \cos (\alpha)\right),
$$

where $\vec{v}_{r e l}=\vec{v}_{1}-\vec{v}_{2}$ (see Fig. 1), $\mu$ is the reduced mass and $\alpha$ the angle between the two velocity vectors. Since we are interested in reducing $E_{\text {coll }}$, we need to find ways to reduce either both velocities in the LFR or the angle $\alpha$. To reach $E_{\text {coll }}=0$ the only viable option is to set $\alpha$ to zero since setting the velocities to zero would result in zero flux. Using two straight beams at low but non-zero angles $\alpha$ can lead to very low collision energies, as was impressively shown by the Bordeaux group [18-20]. Collision energies below $5 \mathrm{~K}$ were obtained by setting one velocity equal to zero, by trapping a sample of $\mathrm{OH}$ radicals, and crossing the trap with a sample of velocity-filtered, cold ammonia molecules [21]. But true zero collision energy is reached, according to equation 1 , by setting $\alpha=0$ and $\left|\vec{v}_{1}\right|=\left|\vec{v}_{2}\right|$. Crucially, this is the case independent of the absolute values of $\left|\vec{v}_{1}\right|$ or $\left|\vec{v}_{2}\right|$ in the LFR.

This is the basis of the merged beam technique that shall be described in the following. This technique has been available for ion-molecule reactions already for several decades [22-25], but the adaptation to neutral reactions required the development of an entirely new toolset which was invented and brought to perfection mainly in the context of deceleration of neutral molecules [1-3]. Indeed, Stark and Zeeman deceleration [26-38] provide all the means required for an exquisite control of the motion of neutral polar or paramagnetic particles in the gas phase.

The merged beam technique requires at least one of the beams to be bent, which presently requires electric or magnetic guides. Guides for neutral particles have been in use in the reaction dynamics community for several decades already, mainly to select a particular rotational state of a molecule [17]. The construction of bent guides was first done in the context of velocity filtering which provides a translationally cold, continuous beam of neutral molecules [39-44].

The neutral merged beam technique was first demonstrated experimentally by Narevicius and coworkers [45] and was independently developed also in our lab [46, 47], and studied theoretically by Wei and Herschbach [48]. Several scientific studies have demonstrated the potential buried in this technique in the past few years [45, 47, 49-53].

This article gives a detailed account of the design and construction of our apparatus. To the best of our knowledge there currently are only two neutral merged-beam machines 
operational, but other efforts are under way $[54,55]$. The version by the Narevicius group and the one described here naturally base on the same principle but they differ in certain key aspects. While we here focus on our own machine, we will outline, wherever possible, the differences with the other apparatus. For details, however, we refer to publications by Narevicius et al.

This review is not meant as a manual to build a merged-beam machine, but rather as a guide-line in designing one. It thus is not crucial to have complete descriptions of the two setups that are presently operational. We also do not give any of the underlying science that is relevant for the understanding of a merged-beam experiment. Our intention is to give a detailed account of our planning and implementation of this setup, hoping that it may serve a young graduate student on his or her way towards a merged beam experiment. For all of the scientific foundations of merged beams we refer the reader to some of the many excellent review articles and books that are available for molecular beams [15], the translational control of gas-phase molecules [1-4, 11,56-58], or reaction dynamics $[16,17,59]$. The level of technical detail at times is quite high, at the risk of providing information that may seem trivial to the experienced experimentalist but that may be vital to a junior scientist in the field. It is our hope to provide a view into our minds as we move from the inception of a new concept (merging neutral molecular beams) to an actual, functioning apparatus. Ideally, one or the other young scientist can extract useful information also for the construction of a similar experimental apparatus.

\section{General experimental considerations}

Because the goal of this fairly complicated experiment is to obtain highly detailed information on complex (chemical) processes it is crucial to carefully select and design the individual components of the apparatus. The key components in this type of setup are the beam sources, the atom/molecule guides, and the detection method. Our goal when developing the merged beam method was to find a way that would allow us to obtain fundamental information on molecular scattering dynamics and to study astrochemically relevant reactions. Of the relevant molecules in interstellar space many are paramagnetic and/or polar. Incidentally, these are the properties that are also required for the guiding of molecules using electric or magnetic fields. Our plan thus became to produce supersonic expansions of the reactants, using pulsed valves and discharge sources, and bend the resulting beams onto a given axis, using magnetic and electric guides. Because we expected the discharge sources to be rather messy and possibly not very selective, we decided to use two guides which would simplify the purification of the beams prior to the collision studies.

In an ideal reaction dynamics experiment we want high count rates, low and controllable translational temperature, tuneable collision energies that cover the full range from zero to thousands of Kelvin, one selected internal state of each reactant, oriented reactants, state-specific detection and full information on the velocity vectors of all reaction products. In reality each of these requirements has been met in some experiment, but to date there is no apparatus that satisfies all these conditions simultaneously. When planning our experiment we had to prioritise these aspects and decide which ones to aim for and which ones to postpone to later iterations of the apparatus. Obviously, the new aspect of the merged-beam experiment is the option to reach $0 \mathrm{~K}$, and in the first version described here this indeed was the only goal (note that we use Kelvin both as a unit 
for temperature and for energy, using the relation $\left.[\mathrm{K}]=[\mathrm{J}] / k_{B}\right)$. Our main requirements were low translational temperature (since this ultimately limits the minimum average collision energy achievable in this experiment), tuneable collision energy that includes zero Kelvin, low internal temperature of both reactants, high density, and a maximum selection of accessible reactions, given the limitations imposed by the other criteria. For our first steps into the territory of merged neutral beams we decided to study a particular class of reactions that would alleviate some of the initial technical difficulties, be ideal systems to characterise the technique, and give access to several fundamentally interesting aspects of reactive collisions: Penning ionisation [60, 61].

In this type of electron transfer reaction a metastable particle $A^{*}$ collides with a particle $B$. If the internal energy of $A^{*}$ exceeds the ionisation energy of $B$ then a reaction according to

$$
A^{*}+B \longrightarrow A+B^{+}+e^{-}
$$

can happen. Because one of the reaction product is an ion it can be readily detected in a time-of-flight mass spectrometer (TOF-MS), thus avoiding difficulties in the detection and reducing the critical planning to source and guides.

The molecular beam source has to be general, produce high density samples with low translational and internal temperatures, and it needs to be compatible with the electric and magnetic guide that will be added later. Several methods have been demonstrated to produce guided molecular beams, and they all have their benefits and shortcomings. As said previously we are using pulsed supersonic expansions, but it nevertheless is worth to also consider other options. In the following paragraphs we will briefly discuss three possibilities: room temperature effusive beams, cryogenic effusive beams with hydrodynamic enhancement, and pulsed supersonic expansions.

Room temperature effusive beams provide continuous flux and are experimentally quite simple to implement and operate. They produce an almost one-dimensional, thermal velocity distribution, and thermally populated internal states. The former is an advantage when used in combination with, e.g., velocity filters because they provide a relatively straightforward way to obtain slow molecules. But in reaction dynamics studies the wide (thermal) velocity distribution mostly renders difficult a precise selection of experimentally probed velocities. Such a source produces continuous beams that always contain all contributing velocities at any given point along the beam axis.

As will be shown below, it is highly beneficial to use a pulsed source instead of a continuous one. They provide the crucial advantage that the packet disperses as it flies through the guide, and the molecules separate by velocity, thus allowing for much higher energy resolution. In a continuous beam this advantage is lost. Furthermore, the high rotational temperature obtained from room-temperature effusive sources means that many rotational states, possibly even vibrationally excited states, contribute to the reaction. Previous experiments have shown that the rotational distribution remains mostly conserved also when such a beam is electrically guided $[43,44]$. Thus, room temperature effusive sources, while simple devices, are not suitable for the present type of experiment.

Instead of operating an effusive source at room temperature it can be combined with buffer gas cooling technologies. In previous studies, such cryogenic effusive sources have been shown to produce, given the right settings, hydrodynamically enhanced, cold molecular beams [62-66]. These beams provide high-density, continuous beams with 
translational and rotational temperatures in the range of a few Kelvin. The advantage is that one has low translational energies straight from the source which could simplify several of the technical aspects described below. The downside, and the reason why we decided against such sources, are the normally continuous character of the beam and the high degree of experimental complexity added by the source alone.

The third option is the use of a supersonic expansion [15]. This type of molecular beam source is most common in spectroscopy and collision experiments and has proven its power and versatility for several decades. These sources produce dense packets of translationally and internally cold molecules or atoms, and they can easily be combined with discharge sources as well. Supersonic expansions can be pulsed or continuous, but for the reasons given below only pulsed sources are considered here. What may at first seem as a drawback of this type of source is the fact that the beams move at very high velocities in the laboratory frame of reference. However, as explained in the introduction, in view of reaching $E_{\text {coll }}=0$ in the merged beam experiment the lab-frame velocity is not relevant. So long as we are able to produce two beams that move at the same speed we are fine (high absolute velocities can, however, affect the resolution in a merged beam experiment [25]).

The pulsed character of these sources leads to an effect that is crucial to reach the high resolution and low temperatures targeted in a merged beam experiment: the dispersion of the beam pulses $[47,67]$. This effect is illustrated in Fig. 2: panel A shows the phase space distribution of the molecular beam at the source (left side in red), and after a certain flight time (right side, in green), respectively. Panel B shows, for comparison, that of a continuous beam. In the pulsed case we can, given an appropriate method of product detection, slice out a very narrow range of the velocities from the beam (for example, by extracting ions in a pulsed time-of-flight mass spectrometer). In Fig. 2a this corresponds to a narrow slice in space, as indicated by the vertical lines spaced by $\Delta x$. The figure immediately shows the advantage of the pulsed source, as well as of a relatively long flight time between the source and the collision region (where the flight time is determined by the beam velocity and the length of the guides, see below). If the flight time is short then a given spatial selection picks a large range of velocities, $\Delta v_{1}$. In contrast, after a certain
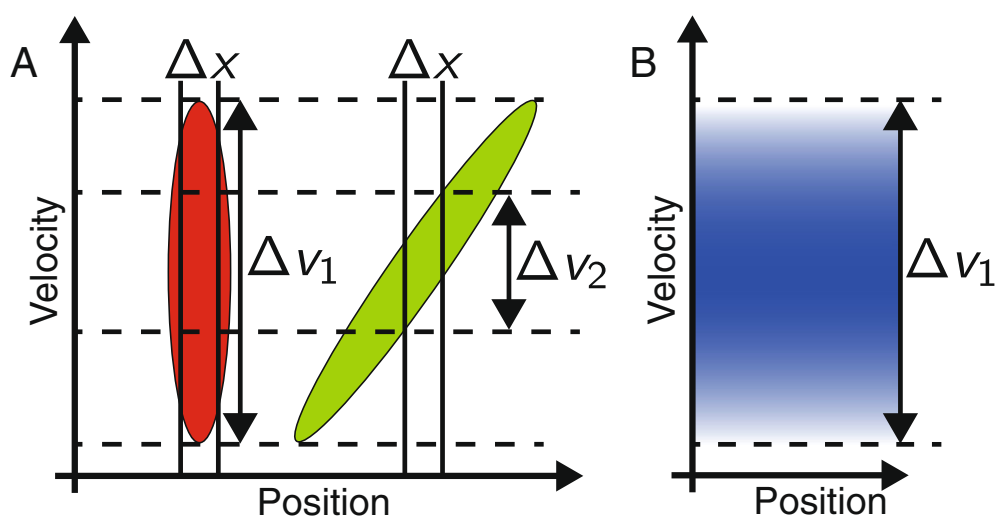

Fig. 2 Beam dispersion. a Phase space distribution of the particles in a molecular packet directly behind the source (left side) and some flight distance down stream (right side). $\Delta x$ is the width of the detection zone, $\Delta v_{i}$ the velocity range that would be probed without $(i=1)$ and with $(i=2)$ the dispersion of the packet during the flight from source to detection. $\mathbf{b}$ phase space distribution of a continuous beam with the same velocity spread as the pulse in panel a 
flight time when the fast and the slow molecules are spatially separated from each other, the same $\Delta x$ only covers a velocity segment $\Delta v_{2}<\Delta v_{1}$. Since there is no cooling involved in this process, the reduction of $\Delta v$ necessarily is accompanied with a loss in number density. But what is evident from these considerations is that one ideally would like to have a beam source that provides pulses as short as possible. Presently available sources, when combined with flight distances of $\approx 1-2 \mathrm{~m}$, provide $\Delta v$ of around $10-20 \mathrm{~m} / \mathrm{s}$, thus defining the resolution and the minimum achievable average collision energy.

A complete, formal discussion of the resolution achieved in a merged beam experiment is beyond the scope of this paper. Much of the relevant concepts has been developed already in the context of ion-molecule reactions in merged beams [25, 68]. Crucial factors are short initial pulses (see above) and high speed ratios, $v / \Delta v$. Long flight times, i.e. long guides or low velocities, further contribute to an improved resolution.

The importance of beam dispersion visualises why the use of continuous sources, while potentially beneficial for higher data acquisition rates, make it hard to reach very low collision energies or high resolution. It should further be noted that in current experiments the ultimate resolution is determined by longitudinal velocities alone. A significant reduction of the longitudinal velocity spread would mean that the resolution would be given by the transverse velocity and velocity spread. In a merged beam experiment, three factors may determine the transverse spread: the geometric selection of the components of the beam that are fed into the guide, the acceptance of the guides themselves, and the geometric selection between the end of the guide and the interaction region. The transverse acceptances of the guides used here are on the order of 10-20 m/s for the present particles. The geometric selection at the end of the guides is given by their radii and that of the interaction region, and by the distance between the two. Having guide diameters of $8 \mathrm{~mm}$ and a radius of the interaction region of $5 \mathrm{~mm}$ we can use the distance of 300 $\mathrm{mm}$ to calculate a ratio of transverse-to-longitudinal velocities of 40-100. At the lowest relative longitudinal velocity this would correspond to transverse velocities up to $10 \mathrm{~m} / \mathrm{s}$. The same value is also obtained in trajectory calculations where a distribution of $\pm 5 \mathrm{~m} / \mathrm{s}$ is found. It has to be noted, however, that the actual distribution in relative transverse velocities is smaller than that because the particles with the extreme transverse velocities in each of the beams will not cross inside the interaction region. Consequently the transverse relative velocity distribution at this point is at least a factor ten narrower than the longitudinal spread and at this point can be neglected in the data interpretation.

Based on the above considerations our molecular beam source of choice presently is the pulsed supersonic expansion. For the study of excited species or radicals it can easily be combined with discharges or other radicals sources. As mentioned above, the downside of pulsed supersonic beams is their very high speed in the laboratory frame. The merged beam technique itself is insensitive to this, but technically it is a crucial factor. Specifically, since we are using guides to bend molecular beams the forward velocity will determine in part the entire geometry of the apparatus.

An important decision indeed is whether to use a single bent guide and combine it with a direct expansion, as was done by Narevicius and co-workers [45], or to use two bent guides as done here [47]. Depending on the target systems these guides can then be electric and/or magnetic guides: guides work only for certain molecules, either paramagnetic or polar, and only with suitable Zeeman or Stark effects. Using only a single guide has the advantage not only of reduced technical complexity but also of increased applicability 
because the choice of one reactant is not limited by the required dipole moment. Two guides, in contrast, increase the purity of the experiment because both reactants need to pass the requirement of guidability. In seeded supersonic expansions this means, for example, that none of the carrier gas reaches the interaction zone. Similarly, in a beam that contains a molecule in different states only those states reach the reaction zone that have a suitable Stark or Zeeman effect [69]. As an example, of the two components of the ammonia tunnelling doublet one is high-field seeking (hfs) while the other is lowfield seeking (lfs). Only the lfs is observed in the reaction. By the same reasoning when using an expansion that contains different molecules only some will be guided while others are eliminated from the beam. This is of particular interest when using radicals that are produced in, for example, a discharge source. Given the right conditions, only one of the fragments formed possesses an electric or magnetic dipole moment that allows it to be guided, while the rest is removed from the beam [69].

As the molecules travel through the guides the force generated by the Stark or Zeeman effect keeps the beams collimated and produces the required centripetal force to keep the particles on a curved trajectory $[39,56]$. High forward velocities lead to strong centrifugal forces. Precisely this effect is used in a velocity filter where the fast molecules are removed because the centripetal force is insufficient [39]. An estimate for the maximum velocity that can be guided in a curved electrostatic guide is obtained by setting the velocity-dependent centrifugal force equal to the guiding force produces via the Stark effect [40,44]. For example, for a symmetric top molecule with no zero-field splitting and a purely linear Stark effect (which is a good approximative description for $\mathrm{ND}_{3}$ ) we find the maximum guidable velocity to be given as

$$
v_{\max }=\sqrt{\frac{2 \mu_{0} F_{\max }}{m} \frac{|M K|}{J(J+1)}\left(1+\frac{R}{r_{0}}\right)},
$$

where $J, K$, and $M$ are the quantum numbers for total angular momentum, its projection on the molecule z-axis, and on the electric field axis, respectively. $\mu_{0}$ is the permanent electric dipole moment of the molecule, $m$ the molecular mass, $F_{\max }$ the maximum electric field in the guide, $R$ the bend radius, and $r_{0}$ the inner radius of the guide. Because in the present experiment we are interested in guiding fast molecules from a supersonic expansion these parameters need to be selected accordingly. Equation 3 shows that, for any given molecule in a particular rotational state, $v_{\max }$ is higher for larger $F_{\max }$, larger $R$, or smaller $r_{0}$. A small $r_{0}$ is inconvenient because for the optimum operation the guide dimensions should be matched with the emittance of the source, i.e., the diameter of the molecular beam when it enters the guide. Electric field strengths $F_{\max }$ with current production techniques for electrodes can not be raised much beyond $200 \mathrm{kV} / \mathrm{cm}$ without a considerable risk of electrical arking. Thus, the best option to increase $v_{\max }$ is a large bend radius $R$.

The same considerations apply to a magnetic guide. Additionally, in this case one has to decide between using permanent magnets (as used here) and electromagnets (as used by Narevicius et al.). Very strong permanent magnets made from $\mathrm{NdFeB}$ are available commercially at very low cost, and they are trivial to "operate". The downside is that a guide built from permanent magnets can neither be switched off, nor adjusted to other experimental parameters. Also, electromagnets potentially are able to produce considerably stronger magnetic fields which widens the range of guidable particles and velocities, 
and allows for a smaller bend radius. Conversely, electromagnets require much additional equipment for the generation, and possibly switching, of high currents, as well as complex setups for cooling of the magnet coils.

The choice of magnetic vs. electric guide depends on the system of interest. Zeeman guides allow for the guiding also of atoms while an electric guide can, if properly operated, be used both for hfs and lfs. [70] hfs states are of particular interest because the absolute ground state of any species is hfs. In particular when investigating larger molecules all of the lower lying states are hfs. These states can be guided by using the alternating gradient approach where ac fields are applied to the guide electrodes [70-72]. If properly constructed and operated the same guide can be used both for hfs and for lfs molecules.

Two guides of the same type are difficult to combine because sending, for example, one beam of polar molecules into an electrostatic guide while the voltages are switched on (which in this case they have to, since they are needed to guide the other beam, and because the desired temporal beam-overlap means that one molecular beam has to be inserted into the other guide while the other molecular beam is at the same point in space) is not possible without considerable losses. As the molecules would approach the guide from the outside they would be repelled by the forces from the inhomogeneous fields, just like they are confined while inside the guide. An option would be Y-shaped guides where no beam has to move into another guide because a single device is used to guide both beams from the source to the merging. These would lead to much reduced losses, but due to limitations by the Liouville theorem the merging nevertheless must lead to either reduced number densities or increased temperature. Two different guides, in contrast, allow for the lossless merging of a purely polar and a purely paramagnetic beam (in fact, one of the beams can be both polar and paramagnetic, while the other one can only have either of the two properties).

An important aspect are possible fluctuations in the densities of the two molecular beams. Not only are pulsed supersonic expansions occasionally unstable and yield fairly important pulse-to-pulse fluctuations, one also needs to consider differences in transmission for different source conditions. To be able to account for these effects one has to install a system to monitor the densities in each of the beams individually and in real-time. These values then need to be used to normalise the observed reaction rates.

\section{Design and construction}

Figure 3 shows an overview of the experimental setup. Before describing the individual parts in detail we outline a few fundamental aspects that needed to be considered. In the previous section we have defined the overall requirements for the setup: we need two supersonic expansions, two guides - one electric and one magnetic - and a system for product detection and beam characterisation. From equation 3 we know that we need a guide with a curvature of roughly $5 \mathrm{~m}$ in order to transport molecules like ammonia at velocities up to $1000 \mathrm{~m} / \mathrm{s}$. As is indicated by the dashed line in Fig. 3 our guides are designed such that there is no direct line of sight from the source to the reaction zone, requiring an $\approx 10^{\circ}$ turn. The resulting guides thus have a length of $\approx 1-2 \mathrm{~m}$.

With technical means available to us it was not possible to produce a bent electrostatic guide from single, bent, electrodes at these dimensions. However, a bent guide can be well approximated by a series of straight segments that are connected at a small angle 


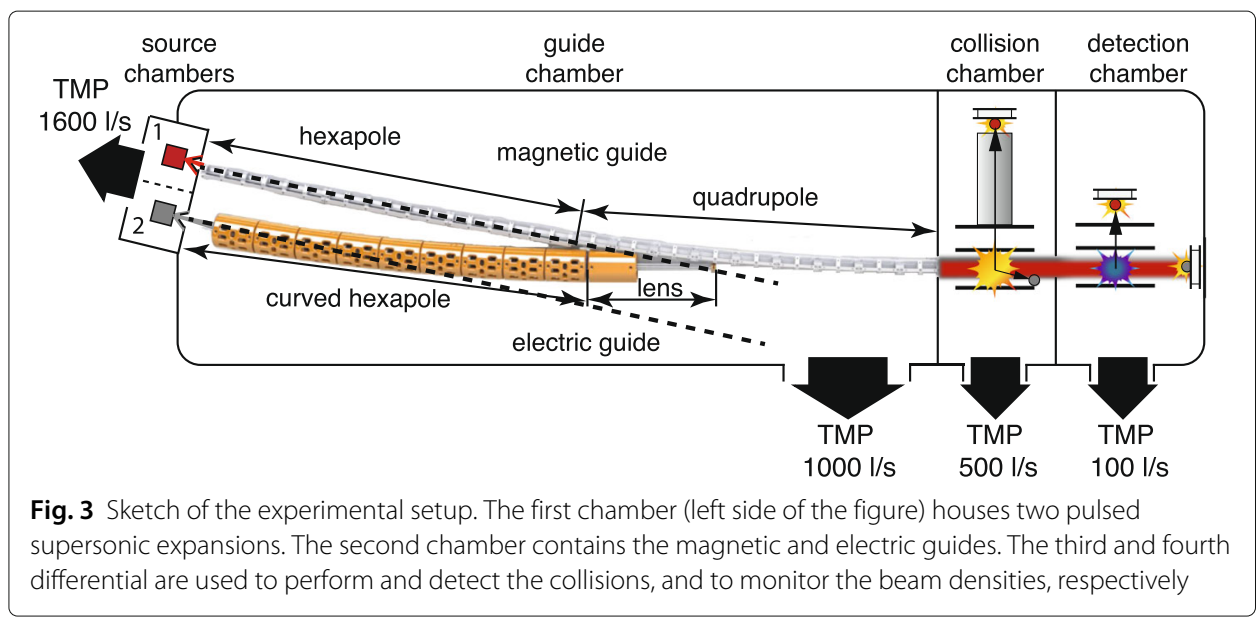

[73]. Our electric guide is composed from multiple straight segments each $100 \mathrm{~mm}$ long and connected with a $1^{\circ}$ tilt angle. Because we decided to build the magnetic guide from permanent magnets instead of electromagnets, a truly curved guide was not realistic, and we decided to use straight segments also here. All of these segments, both electric and magnetic, are mounted on the same base plate, aligned outside the vacuum chamber and then installed. No further adjustments are possible once the guides are in vacuum.

A crucial aspect obviously is the merging of the two beams. In order to achieve this, one beam has to be sent through the other guide. Narevicius et al. use a magnetic quadrupole guide which is designed in a way that is very open on the side. Our magnetic guide is composed of a first region where a hexapole is used, and a second region where an open quadrupole is used that allows the polar beam to be focused through the magnets and onto the paramagnetic beam. The electric guide is built from a series of short segments that serve to guide the beam, and a long segment at the end which is used as an electrostatic lens. Voltage on the lens leads to collimation of the particle beam and focusing through the magnets of the Zeeman guide into the reaction zone.

The entire setup is housed in a four-fold differentially pumped vacuum chamber. The four chambers, described in more detail in the next sections, are a source chamber, a chamber housing the guides, a chamber where the collisions are detected, and a chamber to characterise the two molecular beams. Source and guide chamber are constructed using ISO-F and ISO-K type flanges with Viton or other rubber gaskets. The last two use $\mathrm{CF}$ flanges with copper gaskets. The adapter tube to make the transition between these standards is also the one that provides the kink, which is necessary because of the bend in the guides. A commercial half-nipple with an ISO-F flange was welded to a CF flange with a 10-degree tilt between the flange faces. This separation in two standards was chosen for simplicity (and cost), and because other than continuity there is no reason to use the CF standard on a source chamber where the pressure during operation usually lies above $10^{-5}$ mbar. Our target pressure for the guide and collision chambers was in the range of $10^{-8}$ mbar. Even in that range no significant losses are to be expected from collisions of guided molecules with background gas. Bake-out of the apparatus is not only not required, it also is not possible because the permanent magnets must be expected to start demagnetising at temperatures above $\approx 60^{\circ} \mathrm{C}$. 


\section{Source chamber}

The source chamber, shown on the very left in Fig. 3, is a single squared chamber made from stainless steel in our local workshop. It contains two compartments, separated by a vertical wall, that each houses one supersonic expansion. A single turbo molecular pump $(1600 \mathrm{l} / \mathrm{s})$ is installed on top of the chamber and generates vacuum in both compartments. Cross-talk between the two half-chambers is minimised by closing off the chambers all the way up to the pump. Base pressures in this chamber are in the range of $10^{-7} \mathrm{mbar}$ and rise to $10^{-5}$ mbar during operation. Pressure gauges monitoring the pressure in each compartment demonstrate that the separation wall does lead to a pressure differential, but that nevertheless operating one source also leads to a noticeable pressure increase in the second compartment.

The pulsed valves, along with a skimmer, are each centered and fixed on the same type of mount that can be detached from the chamber and allows for an easy swapping of different types of sources. This module is aligned outside the chamber and then clamped to the inner wall of the source chamber. Alignment pins guarantee that the source module is lined up with the respective guide. The sources can be cooled using liquid nitrogencooled nitrogen gas, and heated using a heating coil, in order to adjust the speed of the expansions.

In the studies performed to date an Even-Lavie valve (ELV) was used for one expansion while a General valve (series 99; GV) was used for the other one. Both can be equipped with an electron impact excitation (EIE) device for the production of radicals or metastable states. As EIE we have used a circular filament mounted between two cylindrical electrodes around the expansion such that electrons can be accelerated with energies of 100-200 V into the expansion. Alternatively the ELV, but not currently the GV, can be equipped with a commercial dielectric barrier discharge [74].

Other pulsed sources would also work for this type of experiment (see, for example, [75]). We had originally intended to use two ELVs to study the $\mathrm{Ne}\left({ }^{3} \mathrm{P}_{2}\right)+\mathrm{NH}_{3}$ Penning ionisation reaction but then had to realise that the kapton gaskets in the ELV do not withstand ammonia for very long and are chemically damaged. The GV, just like several other sources, does not suffer from this problem.

\section{The magnetic guide}

Figure 4 contains all the relevant information about the magnetic guide. Panel A shows the overall shape of the guide, with the source on the left and the interaction region on the right. It is built from several straight segments that are connected with small angles inbetween [47]. The first section of the guide is a hexapole, the second is a quadrupole with a wide opening on the side for easier merging with the polar beam. The magnetic fields are produced by off-the-shelf commercial $\mathrm{NdFeB}$ permanent magnets with a nominal remanence of $1.17 \mathrm{~T}$. The first section is built from three straight hexapole segments with a $1^{\circ}$-tilt between adjacent segments, forming an overall bend of $2^{\circ}$. The second section is constructed from 10 segments that perform an additional $9^{\circ}$-bend. Overall, the magnetic guide has a bend radius of $\approx 6 \mathrm{~m}$.

Figure $4 \mathrm{~b}$ shows a cross section of the first guide section. Each segment is built from eight hexagonal mounts made from magnetic steel. $183 \times 10 \times 100 \mathrm{~mm}^{3}$ magnets are inserted to form a $30 \mathrm{~cm}$ long hexagonal pattern. Each of these magnets has a hole at the center, is held by a screw, and pushed into position by a set screw at each end. The field 


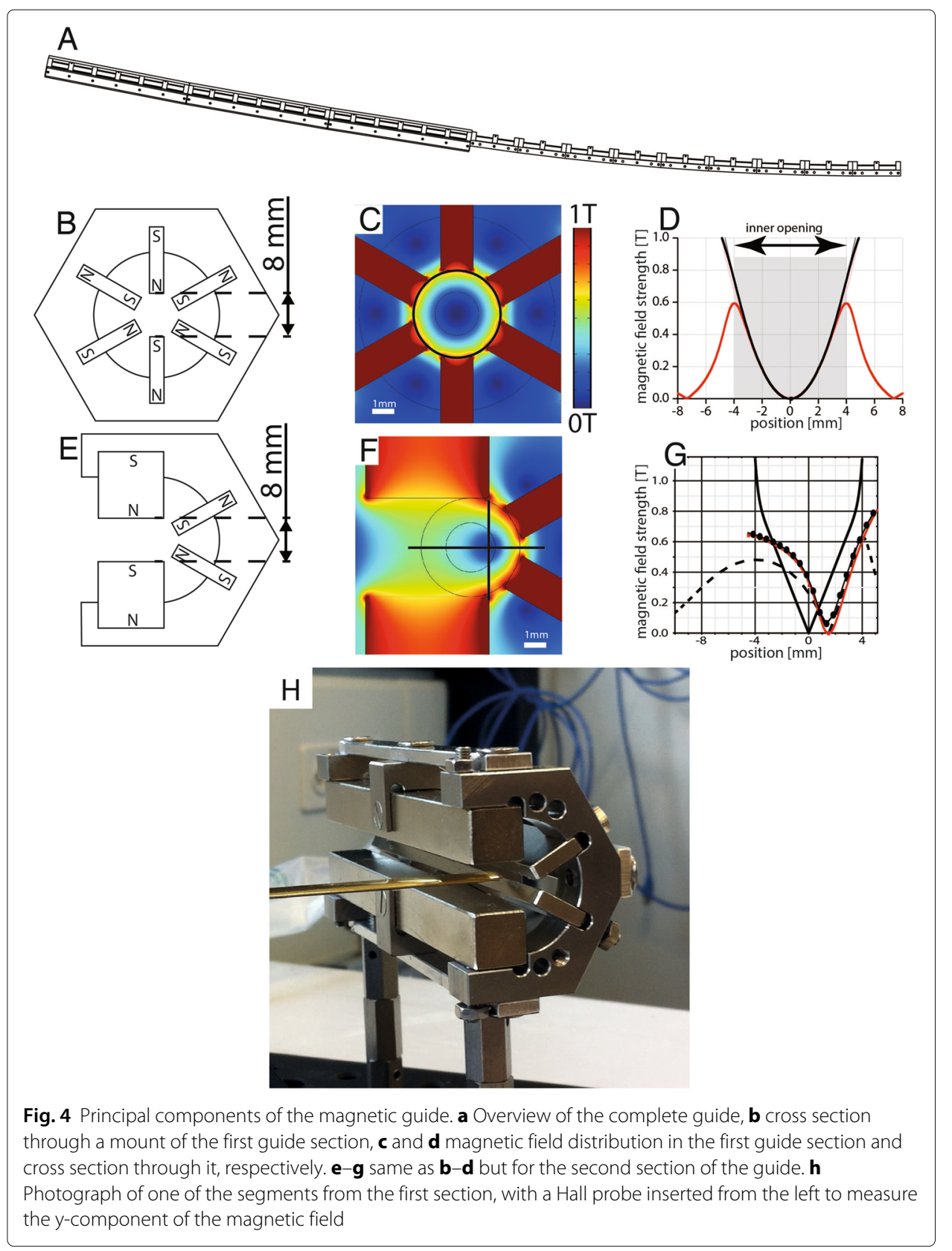

distribution inside the guide is shown in Fig. 4c as calculated using the COMSOL finite elements package [76]. A cross section through Fig. 4c, shown in panel 4d, reveals the expected, harmonic potential with a depth of $\approx 0.6 \mathrm{~T}$.

A segment from the second section is shown in the photograph in panel $4 \mathrm{~h}$. The cross section through such a segment is shown in Fig. 4e. Each of these segments has a length of $10 \mathrm{~cm}$ and is built from two $3 \times 10 \times 100 \mathrm{~mm}^{3}$ magnets and two $10 \times 10 \times 100 \mathrm{~mm}^{3} \mathrm{mag}$ nets. Three mounts, each an open and extended hexagon made from magnetic steel, are used to hold each of the magnets in position. Mounting and alignment are done as in the first section. The calculated magnetic field distribution, and cut through it, are shown in Figs. $4 \mathrm{f}$ and $4 \mathrm{~g}$, respectively. Panel $4 \mathrm{~g}$ shows a vertical cross section as solid line, and a 
horizontal cross section as a dashed line, respectively. These graphs show that the geometry chosen here leads to a somewhat reduced depth of the field in the plane of the bend. Towards the outside of the bend the depth is $0.45 \mathrm{~T}$ while vertically and towards the inside of the bend the depth is similar to the hexapole guide. The y-component of the magnetic field in these segments was measured using a Hall probe as shown in the photograph in panel 4h, and it is shown as dots in panel 4g. The field measurements showed that the effective fields were slightly lower than the calculated ones, presumably from a slightly lower-than-specified remanent field on the magnets. The shape of the measured field distribution is closely reproduced by the calculations, thus permitting an accurate quantitative description of the true fields by scaling the calculated fields by a constant factor. When performing trajectory calculations (see below) this is a very important aspect.

\section{The electric guide}

The main information regarding the electric hexapole guide is collected in Fig. 5. Panel 5a shows a top view of the complete guide. It has a total length of $1300 \mathrm{~mm}$ and is composed of 9 straight, $100 \mathrm{~mm}$-long segments and a last, $300 \mathrm{~mm}$-long element. The first 9 segments are the main guide while the last segment serves as the lens for the polar beam. All 10 segments are connected with a $1^{\circ}$-tilt angle between adjacent elements to perform a total turn of $9^{\circ}$. The first 9 elements are connected to two high-voltage power supplies while the lens is operated by two separate high voltage sources.

Panel 5b shows a detailed view of one segment. The electrodes are made from polished stainless steel and inserted into a polyether ether ketone (PEEK) tube. The front view, panel 5b.1, shows the hexagonal symmetry with six 4 mm-rods used as

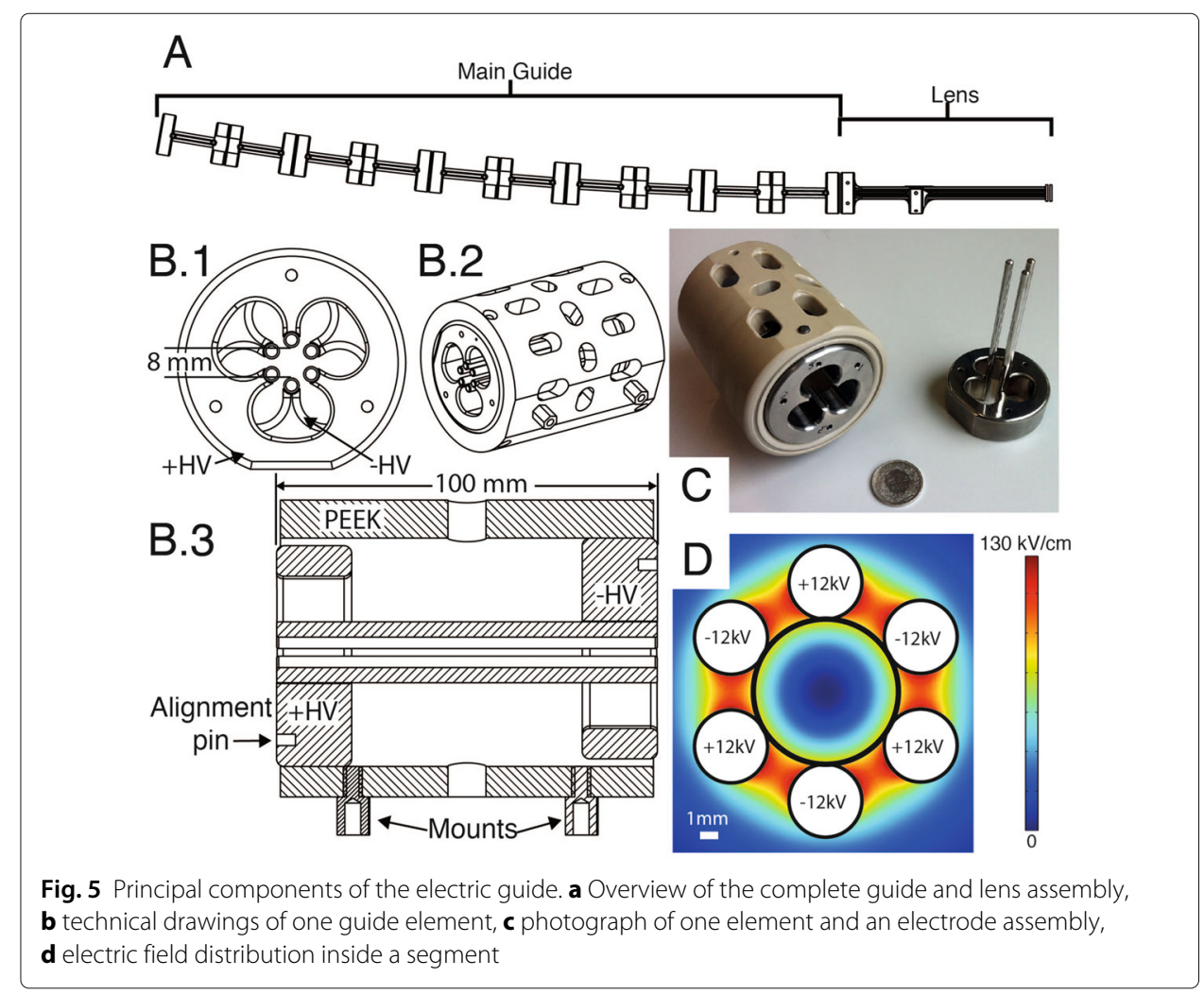


the main electrodes. They are mounted in two groups of three electrodes on individual stainless steel mounts. Panel $5 \mathrm{~b} .3$ shows a cross section through the segment from which it can be seen how the two mounts are inserted into a PEEK tube that serves to electrically isolate the high voltages and provide mechanical rigidity. Stainless steel is shown by top-right to bottom-left hatching. The remaining material is the PEEK tube. The two mounts for the electrodes, seen on the left and right of the sketch, are produced from stainless steel and manually polished to a mirror-finish, using ultra fine grain diamond paste. The electrode rods are made from hardened stainless steel, manually polished and glued to the main mounts using conductive, vacuum-compatible epoxy glue. The electrode mounts are machined to snuggly fit into the PEEK cylinders, and they are held in place by two metal pins inserted from the top and bottom, respectively. One of these pins is also used for the electrical connections.

Further reduction of the risk of discharges to ground is achieved by adding grooves to the PEEK tubes around the metal support rods (bottom of Panel 5b.3). The PEEK cylinders are perforated on one side for more efficient pumping and rapid removal of all non-guided gas during operation. Solid walls are retained on the side facing the magnetic guide in order to suppress collisions with molecules that exit the magnetic guide before the merging takes place. Adjacent elements are aligned by horizontal pins in the electrode mounts (sides of Panel 5b.3). Figure 5c shows a photograph of a finished guide element (left) and one electrode set (right). The coin is a 2-Frank piece for size comparison (approximately the size of a 2-Euro piece or a US quarter).

The lens has a basic construction similar to the short segments, but the electrodes need to extend beyond the mount on one side to bring the magnetic guide as close as possible to the electric guide. Since these electrodes are $300 \mathrm{~mm}$ long it is necessary to provide additional support at the end of the segment. Two PEEK plates are used to which each of the two groups of electrodes are attached via M1 screws inserted along the electrode axes.

Figure 5d shows the calculated, using COMSOL, electric field distribution for a hexapole guide of the present dimensions. Voltages of $\pm 12 \mathrm{kV}$ on the electrodes produce a field distribution that is approximately $120 \mathrm{kV} / \mathrm{cm}$ deep and, for a molecule with a linear Stark shift, almost perfectly harmonic.

It is worth pointing out that PEEK turned out not to be the ideal material for this type of application. While it does have the desired low outgassing rate and and high breakdown voltage (as well as, of course, all the advantageous mechanical properties), is relatively cheap and easy to machine, it turned out that the electrical properties degrade over time. Specifically, after a few months of continuous operation we start to observe a current that in time becomes too high to be able to run the machine. It turned out that this current is mostly on the surface, hinting to surface contaminations. But we found that a simple cleaning of the PEEK does not remove the current completely. Instead, $\approx 0.1 \mathrm{~mm}$ material have to be removed both on the inside and the outside of the tube. Since this is a very cumbersome procedure the material choice here turned out to have been suboptimal. Better choices, from an electrical point of view, would undoubtedly have been Macor or alumina, but these come with the associated price increase and major difficulties in machining.

The two guides combined and mounted on the base plate are shown in the rendering in Fig. 6. 


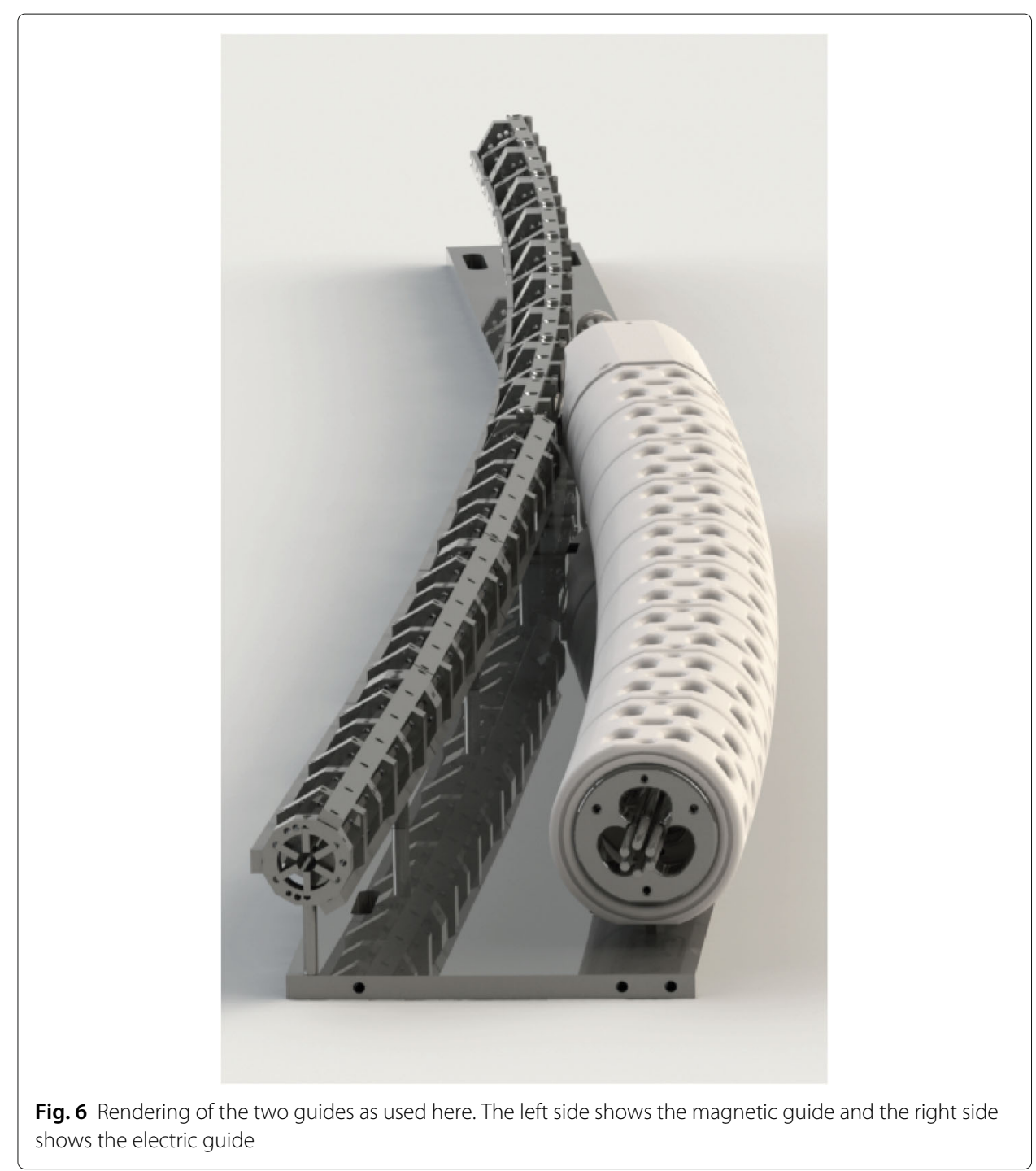

\section{Online measurement of beam densities}

In an experiment we count the number of reaction products formed per gas pulse. For a generic reaction $\mathrm{A}+\mathrm{B} \rightarrow \mathrm{C}$, this reaction rate can be converted to a rate coefficient using the relation

$$
\frac{d N_{C}}{d t}=k N_{A} N_{B}
$$

where $\mathrm{N}_{i}$ is the number density of species $i$ and $k$ is the rate coefficient. Ideally one would like to measure absolute reaction rate coefficients and cross sections. This would, however, require the knowledge of absolute number densities and geometric beam overlap which are highly challenging to accurately measure. Consequently, most experiments provide relative cross sections. But this still requires knowing relative number densities within each of the molecular beams. In a pulsed experiment it is crucial to know how the density in one pulse relates to the density in the previous pulse. We have installed two detectors that allow us to measure the densities in both beams, on an arbitrary scale, at 
each experimental cycle, and to determine a scaling factor to bring all data points on the same scale.

The right side of the sketch in Fig. 3 shows the chamber at the end of the experimental setup that contains two micro channel plate (MCP) detectors. One is on axis with the molecular beams and serves to detect particles that require no additional excitation, for example metastable atoms or molecules. These have sufficient internal energy to launch an amplification cascade in the MCP as they hit the surface. Consequently, an entire pulse can be observed at once, and the relative number density is obtained by reading the amplitude at a particular time. The second detector is mounted off-axis from the beam and serves to detect particles by, for example, resonance-enhanced multi photon ionisation (REMPI). Ions are extracted from the beam and accelerated onto an MCP without mass-separation. For some reactants no efficient REMPI scheme is known, and we have reverted to adding a small quantity of ammonia to the expansion. Ammonia is guided efficiently and easily probed by REMPI. If a certain gas mixture is used for an entire set of measurements then it is safe to assume that at any relative velocity the ratio of ammonia to the desired molecule also remains constant, because no experimental parameter that affects the polar beam has been changed. Thus, knowledge of the ammonia density is sufficient for the normalisation.

Detection by REMPI naturally introduces the laser power $P$ that itself can also fluctuate, and the REMPI signal must be normalised to $P$. For the appropriate scaling we use the expression

$$
S=A P^{p} N
$$

where $S$ is the measured ion signal, $A$ is a scaling factor that contains the ionisation cross section and geometric cross section of the laser beam, and $N$ is the particle number density. $p$ is the effective power dependence that can, depending on the saturation conditions, take almost any value smaller than or equal to the number of photons required for the particular REMPI process. It needs to be determined separately, for the particular laser power and focusing conditions used in every experiment. Unless $p=1$ the normalisation is non-linear and must be done on a shot-per-shot basis. This is because the non-linear

relation between $S$ and $P$ means that $\overline{\left(\frac{S}{P^{p}}\right)} \neq \frac{\bar{S}}{\bar{P}^{p}}$ (where $\bar{x}=1 / M \sum_{m=1}^{M} x_{m}$ for $M$ cycles and $x=S$ or $P$ ). If this is taken into account then knowing $p$ allows for the normalisation, at every shot, of the measured signal and used to extract a relative density $N$.

\section{Reaction chamber and product detection}

While this chamber is where the scientifically most important action is taking place, it in fact is technically the simplest. All it contains is a time-of-flight mass spectrometer (TOFMS) that can be switched on, using a fast high-voltage switch, when reaction products are to be detected. The TOF-MS is positioned such that the beams cross it at the point where they are already completely merged. In favourable cases the reaction product is an ion that can be detected without further steps. In other cases where neutral products are formed they must first be ionised which can be done, for example, by REMPI.

When the reaction products are ions the detection efficiency is higher because REMPI only ionises a small part of the products. Furthermore, any reaction product that is formed outside the TOF extraction region is easily eliminated using a dc-electric field that deflect unwanted ions. 
We note that in our collision chamber we did not install any magnetic shielding because we expected external magnetic fields not to be crucial when detecting ions. It turned out, however, that the atoms emerging from our magnetic guide are strongly oriented [77]. Clearly, a magnetic field in the collision zone is responsible for this, and it currently is not clear what its source is, and wether magnetic shielding would help.

\section{Theoretical characterization}

This section gives an overview on the results from trajectory calculations for the guides described in the previous section. It is worth pointing out that most of these calculations were done prior to the final design and construction of the apparatus. They are essential for the ability to predict the precise properties of such devices, and to ensure that an experiment will function as intended.

\section{Trajectory simulations}

The design of equipment to manipulate the motion of polar or paramagnetic particles in the gas phase is a complex task that requires careful planning. Even though the basic kinematics are very well understood it is far from trivial to predict the motion of an individual molecule inside a guide, let alone the velocity-dependent transmission of an entire thermal ensemble of molecules, even through a passive device like a guide [43, 44, 78-80]. In the simplest cases of straight or bent multipole guides fabricated from single-piece electrodes (or magnets) the transmission can be estimated based on simple formulae like equation 3. In more complex cases like Stark or Zeeman decelerators an accurate prediction without detailed calculations of particle trajectories is nearly impossible.

Segmented guides as used here behave, if properly operated, just like guides formed from continuous, bent electrodes [73]. Because the guide used here really is a section of a polygon, however, unexpected dynamics at the points where segments are joined may alter the velocity-dependent transmission substantially and lead to so-called stop bands [81]. As is well known from accelerator physics these may arise when at certain points in the device the fields are different from the remainder of the device. In this case there is a dependence of the transmission on the transverse oscillation frequency and phase of the particles. Particles oscillating in the guide at some particular frequency and wavelength can have an increased probability to be lost and thus have a lower overall transmission probability.

An accurate and quantitative prediction of stop bands is possible only by calculating the trajectories of large numbers of particles with different initial positions in phase space. Prior to actually producing the two guides described in the last sections we thus have performed detailed simulations for different guide geometries. Work in the field Starkdeceleration from the past 15 years has demonstrated that trajectory calculations are highly reliable in predicting the properties of such devices [1,34], and they thus also allow us to confidently fabricate a device based on simulations.

For our calculations we have used a home written code that numerically solves the equations of motion in the presence of magnetic or electric fields [46], which were calculated using COMSOL [76]. Most calculations were performed using two-dimensional fields, thus implicitly assuming perfect translational symmetry along the main axes of the guides. In particular at the entrance and exit of each guide this translational symmetry does not exist. Explicit, more detailed simulations were performed to ensure that this 
nevertheless does not dramatically affect the transmission. The translational symmetry may also be broken at the joints of two segments, and this was included as required, and detailed below, for the two guides.

\section{Magnetic guide}

Because the magnetic guide is built using off-the-shelf commercial permanent magnets that come in fixed lengths, the joining of straight segments leads to a small gap on the outside of the bend. This gap was included in the calculations by assuming very small regions with reduced magnetic fields. The presence of these gaps leads to stop-bands in the transmission of paramagnetic particles through the Zeeman guide.

As an example, Fig. 7 shows the calculated transmission for metastable $\mathrm{Ne}\left({ }^{3} \mathrm{P}_{2}\right)$ with a flat initial distribution of forward velocities. The transverse velocity distribution was a $\cos ^{2}$ distribution wide enough to cover the entire transverse acceptance of the guide. The two traces show the transmission for the $M=1$ (black line) and the $M=2$ (red line) components, respectively. A stop band is clearly visible in each trace and indeed leads to a local reduction of the transmission to zero. Its position is different for the two $M$ values because the effective magnetic moment is different. Because the permanent magnets do not allow for dynamic adjustment of the transmission curve (as opposed to the electrostatic guide, see below) it was crucial to select the dimensions such that these stop bands would lie outside of the region of interest for our experiments. Our intention was to prepare the paramagnetic particles from supersonic expansions with neon, krypton or argon as carrier gases, in a temperature range of $\approx 100-350 \mathrm{~K}$. The velocity of Neon at these source temperatures lies in the range $\approx 400-900 \mathrm{~m} / \mathrm{s}[15]$ which means that at the highest temperatures it can not be guided anymore. For most of the accessible velocities, however, the stop bands are not a problem because they lie outside the covered range.

The guiding probability is determined by the ratio of mass to magnetic moment, $m / \mu$. Figure 8 shows the transmission (as color code) as a function of $m / \mu$ and of velocity. Horizontal lines correspond to particular rare-gas atoms in their lowest metastable state and the highest corresponding $M$ state. Open (filled) circles represent the expected velocity with a source operated at $100 \mathrm{~K}(300 \mathrm{~K})$. The stop band is seen as a white (corresponding to zero transmission) band curving from $\approx 900 \mathrm{~m} / \mathrm{s}$ at the lowest $m / \mu$ value to lower velocities as $m / \mu$ is increased.

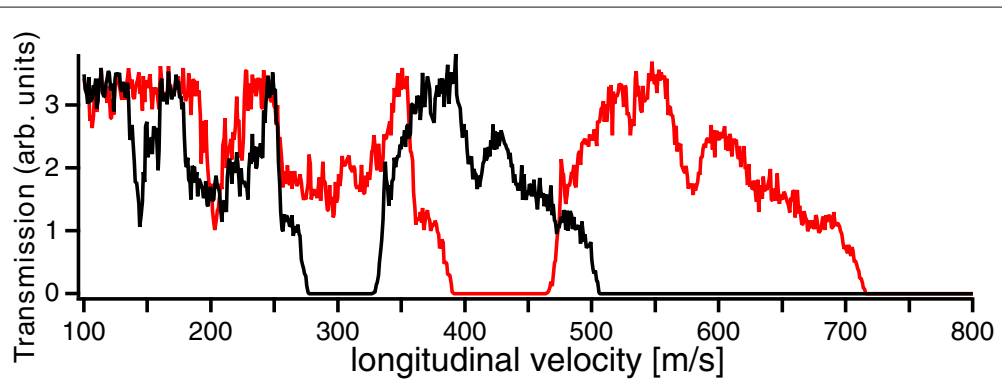

Fig. 7 Calculated transmission for an initially flat velocity distribution of $\mathrm{Ne}\left({ }^{3} \mathrm{P}_{2}, M=2\right)$ (red) and $M=1$ (black) 


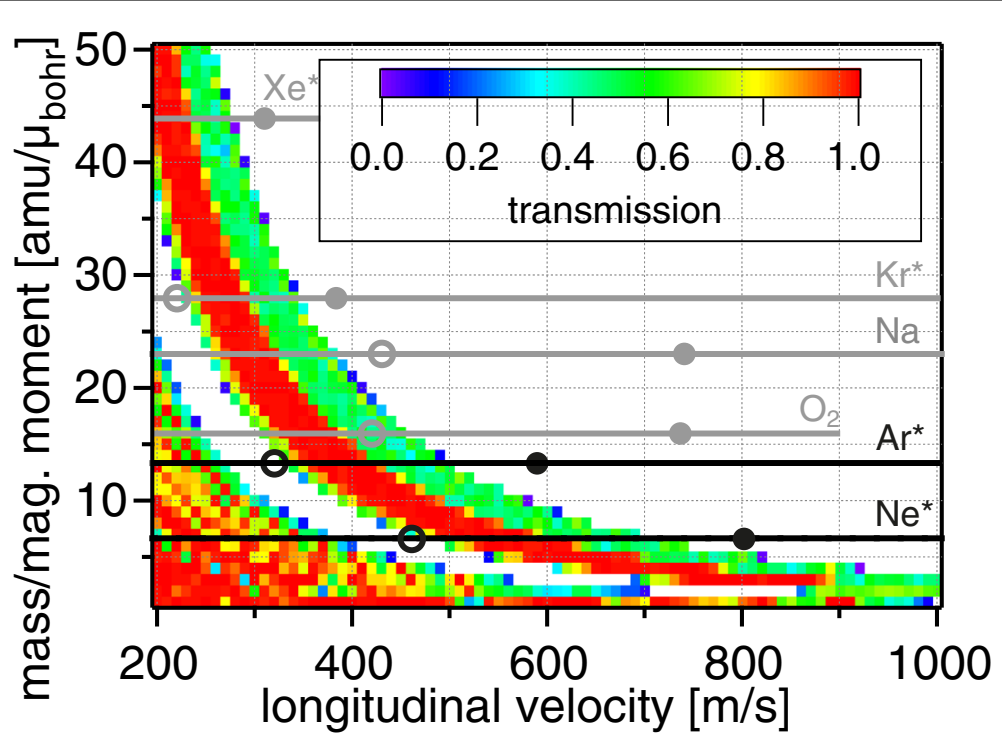

Fig. 8 Calculated transmission probabilities through the magnetic guide as a function of $m / \mu$ and velocity. Horizontal lines show several metastable rare gas atoms and molecular oxygen. In each case the solid (open) point indicates the expected beam velocity for a supersonic expansion operated at $100 \mathrm{~K}$ (300 K)

\section{Electrostatic guide}

No stop bands are expected in the transmission through the electrostatic guide. Even though it is built from several segments, like the magnetic guide, these segments are custom made in our local workshop and were designed such that no gaps exist at the joints of two segments. To achieve this, the stainless steel electrode mounts are constructed with $0.5^{\circ}$ tilts on their outer surface (see Fig. 5). Stop bands might nevertheless occur because of the $1^{\circ}$ angles between adjacent segments, but they are not observed in the calculated transmission curves.

Figure 9 shows the transmission of $\mathrm{ND}_{3}$ in the $(J, K, M K)=(1,1,-1)$ state through the electrostatic guide for different voltages on the electrodes. The black and grey curves in Fig. 9 show the transmission up to the beginning of the lens, when using $\pm 12 \mathrm{kV}$ and $\pm 8 \mathrm{kV}$ on the guide electrodes, respectively. The red and pink curves show the total transmission into the detection region for $\pm 12 \mathrm{kV}$ on the main guide but $\pm 2 \mathrm{kV}$ and \pm 4

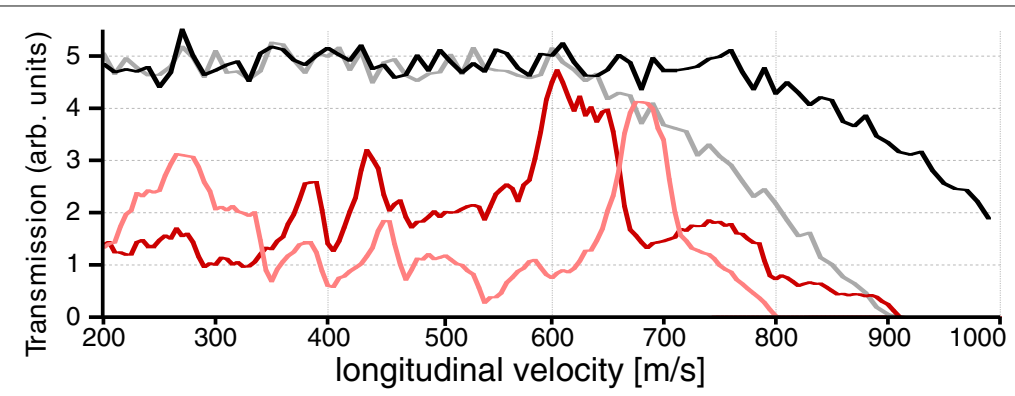

Fig. 9 Calculated ammonia transmission for an initially flat distribution and $\mathrm{NH}_{3}$ in the $(J, K, M K)=(1,1,-1)$ state. The black and grey curve show the transmission up to the lens for $\pm 8 \mathrm{kV}$ (gray) and $\pm 12 \mathrm{kV}$ (black) on the electrodes. Total transmission, including the lens, with $\pm 12 \mathrm{kV}$ on the guide electrodes are shown as red curve (lens voltage $\pm 2 \mathrm{kV}$ ) and pink curve (lens voltage $\pm 4 \mathrm{kV}$ ) 
$\mathrm{kV}$ on the lens electrodes, respectively. The flat initial velocity distribution translates into an almost flat distribution through the first part of the guide with a cut-off velocity that is roughly given by equation 3 . A very different transmission pattern results, however, once the lens is added. In this case the focusing is optimal only for a relatively narrow range of velocities which depends on the lens voltage. Because the distance between the end of the lens and the detection volume is $70 \mathrm{~cm}$ only a well-collimated beam is accepted. The very pronounced structure in the transmission profile can easily be adjusted to match the velocity distribution obtained for a particular molecule, or to match its mass-to-dipole moment ratio, simply by changing the voltage on the lens.

Figure 10 shows the transmission through the electric guide (as color code, without the lens) as a function of velocity and the ratio $m / \mu_{0}$ when the guide is operated with $\pm 12 \mathrm{kV}$. Selected viable molecules are indicated by horizontal lines. In each case the component with the highest effective dipole moment of the lowest guidable rotational state is shown. These results show that the selected geometry, detailed above, indeed provides the required transmission for many small polar molecules in velocity ranges that are easily reached in supersonic expansions with different carrier gases and temperatures. It is important to note, however, that knowledge of $m / \mu_{0}$ alone does not permit a direct estimate of the transmission because the data shown in Fig. 10 has been calculated for a purely linear Stark effect only and only provides a very rough estimate.

\section{Experimental characterization}

Since the main focus of this article is on the design and construction of our merged beam apparatus we will only briefly discuss the operation. Results obtained from this machine have been published elsewhere [3, 46, 47, 51-53, 69, 77]. In the present article we only point out some effects that are important when operating such a device.

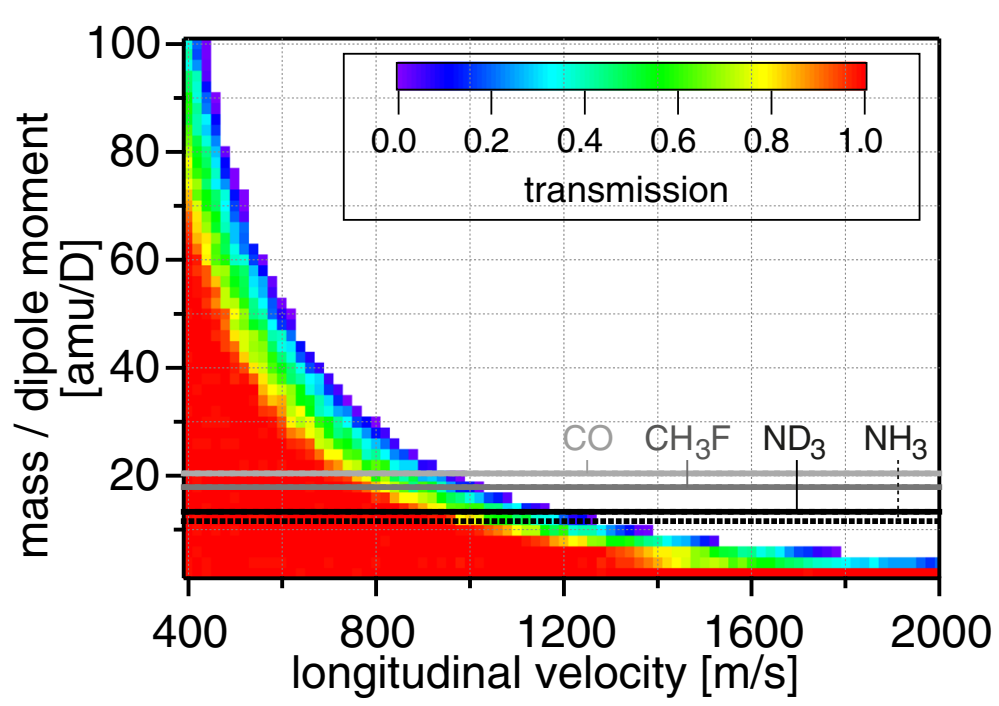

Fig. 10 Calculated transmission probability through the electric guide as a function of $m / \mu_{0}$ and particle velocity. Horizontal lines show several molecules of interest 
We have described above how we measure individual beam densities on a shot-pershot basis to obtain valid reaction rate coefficients. A reliable estimate of the absolute densities in the interaction zone, however, is very difficult. One could hope that trajectory calculations can be used in order to determine the transmission through the guides, but unfortunately also these do not yield values that are accurate to better than one or two orders of magnitude. The main difficulty comes from the ill-defined initial transverse distributions, and the fraction of the molecules exiting the pulsed valve that actually is accepted by the guide, and stably transported to the reaction zone. For this reason we do not provide estimates based on our calculations. One can, however, obtain a rough estimate of the densities from experimental data. Figure 11a shows a mass spectrum of reaction products when merging a beam of $\mathrm{Ne}\left({ }^{3} P_{2}\right)$ with $\mathrm{ND}_{3}$. The three peaks correspond to Penning ionization products $\mathrm{ND}_{3}^{+}\left(20 \mathrm{amu}\right.$ ), and $\mathrm{ND}_{2}^{+}$and $\mathrm{H}_{2} \mathrm{O}^{+}$(both 18 $\mathrm{amu}$ ), respectively. The $\mathrm{H}_{2} \mathrm{O}^{+}$is formed through reactive collisions with background gas. The similar magnitude of these peaks provides an estimate, albeit a very rough one, of the ammonia beam density. By assuming the background pressure in the vacuum chamber to stem entirely from water, similar reaction cross sections, and by neglecting the different velocity distributions, we can assume the $\mathrm{ND}_{3}$ density to be comparable

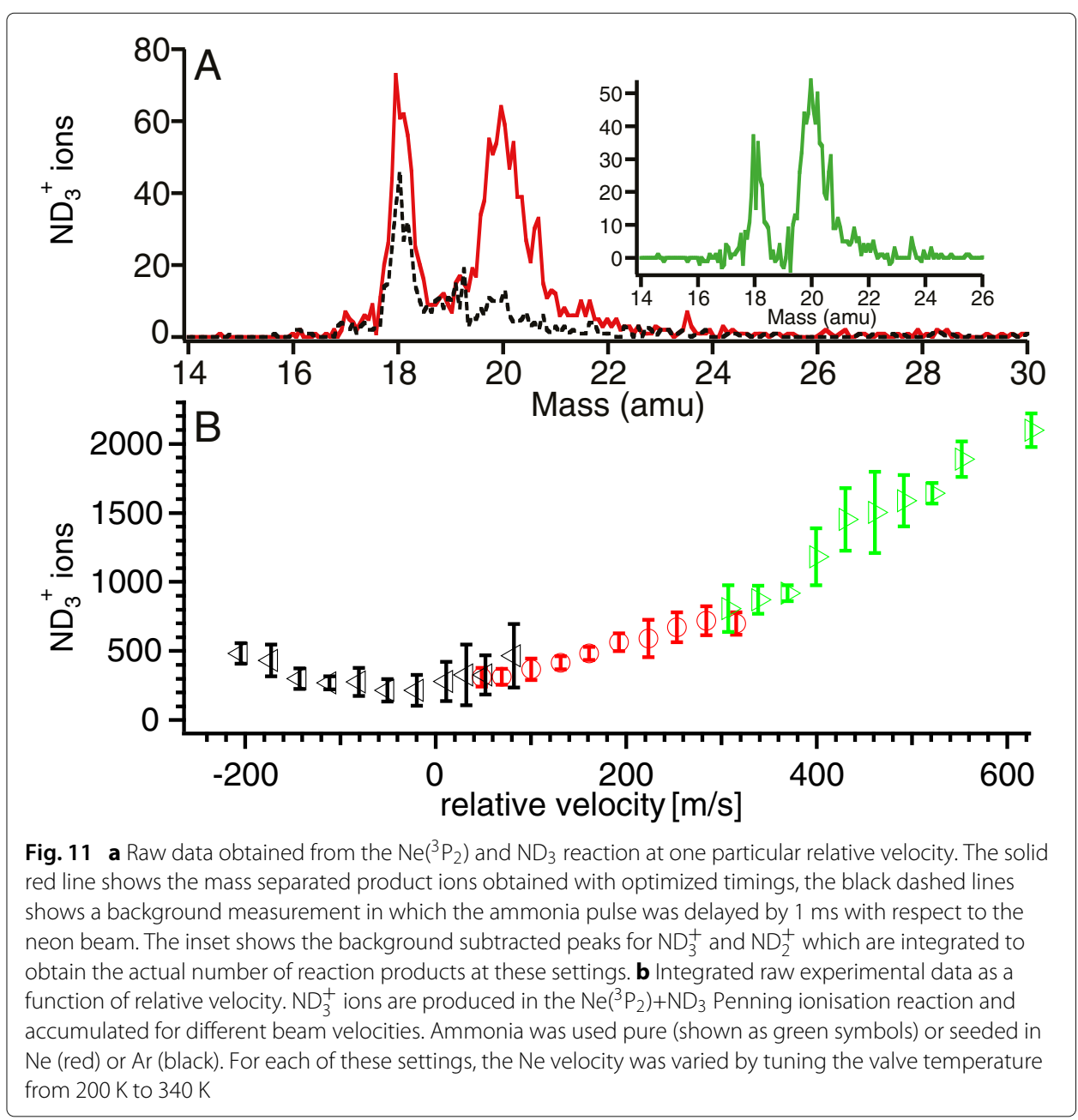


to the $\mathrm{H}_{2} \mathrm{O}$ density which is measured, with a pressure gauge, to be on the order of $10^{8} \mathrm{~cm}^{-3}$.

An alternative route to estimate the particle density is by comparison of the calculated and experimental reaction rate coefficients. Count rates in this experiment require accumulation and counting of individual ions for roughly 5000 molecular beam pulses which yields a total of approximately 1000 product ions. The total measurement time thus is roughly $\Delta t=5 \cdot 10^{3} \cdot L / v$ where $L=2 \mathrm{~cm}$ is the length of the relevant interaction zone and $v=1000 \mathrm{~m} / \mathrm{s}$ is the absolute beam velocity. $L$ is chosen as the approximate distance the particles fly inside the extraction region of the TOF-MS because only those ions are detected that are formed there. This means the relevant, total $\Delta t \approx 0.1 \mathrm{~s}$, and the observed rate is thus about $10000 \mathrm{~s}^{-1}$. Because

$$
\frac{\Delta I}{\Delta t}=k N_{N e} N_{N D_{3}}
$$

we can use this to estimate combined density of both reactants: dividing the measured rate by the rate coefficient, which for the present system has been calculated to be around $10^{-10} \mathrm{~cm}^{3} / \mathrm{s}$ yields the product of the two beam densities, $N_{N e} N_{N D_{3}}=10^{14}$. This number carries a large error mainly because the reaction zone, quantified via the value of $L$, is very difficult to define.

Measuring the geometric overlap of the beams is more complicated and can not be done in real time. In fact, also here it is exceedingly difficult to get an absolute measure and one relies on relative numbers. At first sight one might suspect that the overlap of the two beams is not a function of any of the variables in the present experiment, in particular not of the forward velocity.

This is not entirely true, and to emphasise why it can indeed become an issue, Fig. 11b shows the data we have obtained during our study of the $\mathrm{Ne}\left({ }^{3} \mathrm{P}_{2}\right)+\mathrm{ND}_{3}$ reaction. In this figure, the entire range of relative velocities is shown, reaching from $v_{r e l}=-220 \mathrm{~m} / \mathrm{s}$ to $v_{\text {rel }}=620 \mathrm{~m} / \mathrm{s}$, where $v_{\text {rel }}<0$ means that Neon is faster than ammonia.

Each data point was obtained by accumulating reaction products at a particular relative velocity, and separating them in the TOF-MS. Raw data from one set of velocities are shown in Fig. 11a. The red trace is recorded when the timing of the two valves is optimized to obtain overlapping beams, the black dashed line is a background measurement where the ammonia beam was delayed by $1 \mathrm{~ms}$ with respect to the neon beam. The red trace shows a peak from $\mathrm{ND}_{3}^{+}$and one from $\mathrm{ND}_{2}^{+}$and water, while in the background measurement only the water peak remains. The background subtracted reaction signal is shown in the inset, and a data point for a measurement like that shown in $11 \mathrm{~b}$ is obtained by integrating the mass peaks in this type of data set.

The complete range of relative velocities shown in Fig. 11b was covered by changing the velocities of both beams: ammonia was used pure (green triangles), seeded in neon (red circles), and seeded in argon (black triangles). The different carrier gases provide three different velocities of the ammonia beam, and to fine-tune the relative velocity we then scanned the temperature of the neon valve between $200 \mathrm{~K}$ and $340 \mathrm{~K}$, leading to the different data points for each color. Conditions were chosen such that there is overlap between pairs of velocity ranges: operation of the neon valve at $200 \mathrm{~K}$ and seeding ammonia in neon gives similar relative velocities as operation of the neon valve at $340 \mathrm{~K}$ and using an unseeded ammonia expansion. But when measuring the reaction rate at the same relative velocity, but with different LFR velocities, the values did not automatically match 
and were different by a factor of up to 2 . Because there was enough overlap one could, in principle, have scaled them by hand to match the different ranges at the points of overlap. More reliable data are obtained, however, by considering the physical reason for the mismatch and experimentally compensate for it. We identified the reason as a change in beam overlap when changing the velocity of the $\mathrm{ND}_{3}$ beam.

Figure 12 explains the underlying effect. It shows the end of the electric guide (the lens) on the left, the collision zone in the center, and the density normalisation region on the right. Our density normalisation relies on the fact that the fraction of molecules that pass through the interaction zone and reach the normalisation chamber is constant, i.e., the ratio of the densities measured in the two zones is always the same. Figure 12a covers the range of beam paths in the ideal case where this requirement would indeed be fulfilled, the lens is completely filled and the molecular beam is focused through the detection region and into the normalisation zone while always retaining cylindrical symmetry. Reality, however, resembles more closely the situation shown in panel B for one velocity, and panel $\mathrm{C}$ for another one. In both cases sketched here the overlap with the paramagnetic beam in the TOF-MS is similar, but the fraction of particles reaching the last chamber is larger in panel $\mathrm{C}$ than in panel B. As a consequence, the reaction signal in panel $\mathrm{C}$ would be underestimated with respect to panel $\mathrm{B}$.

The reason for this difference are the particle dynamics in the curved section of the guide and in the lens. Due to the centrifugal force the particles oscillate transversely around a radius that is offset from the geometric center of the guide [56]. As a consequence, the molecules enter the lens off center and are not focused symmetrically into the collision zone. Because they traverse that zone at a different angle they also enter the normalisation chamber differently, and because the entrance to that last chamber is a 1 $\mathrm{cm}$-diameter hole, and the flight distances are $70 \mathrm{~cm}$ (lens-collision zone) and $30 \mathrm{~cm}$ (collision zone-normalisation), different fractions of the beam reach the normalisation zone when the velocity is changed. The normalisation does not provide correct values unless this effect is taken into account.

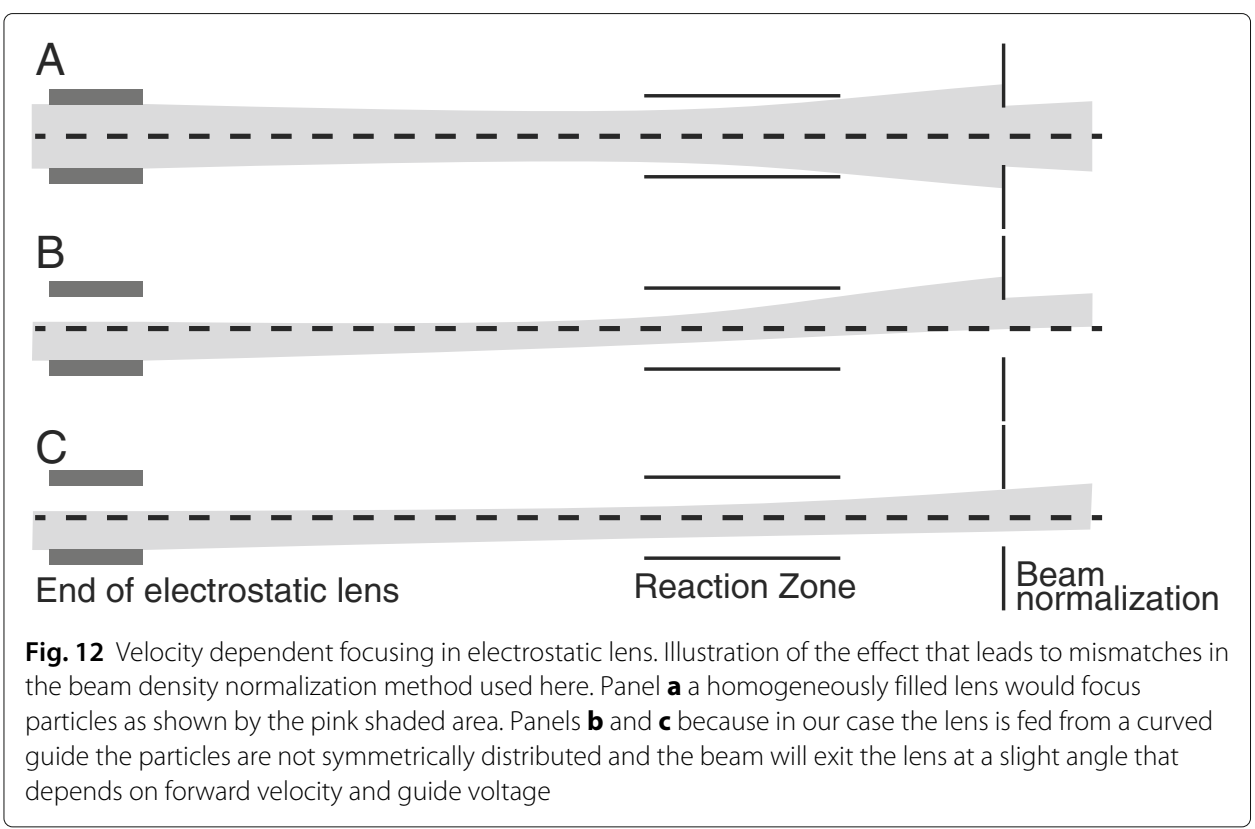


Figure 13 shows the measured transmission of $\mathrm{NH}_{3}$ through the guide and into the last chamber as a function of the voltage on the lens. The top panel shows the ion signal recorded for the $\mathrm{Ne}^{*}+\mathrm{NH}_{3}$ reaction (red circles, left scale) and the $\mathrm{NH}_{3}$ REMPI signal (black circles, right scale) as a function of the lens voltage. The bottom panel shows the ratio between the two signals. Because of the effect described above the maximum in the REMPI and in the ion signals are not at the same lens voltage, and hence the ratio goes through a minimum. The forward velocity-dependent dynamics in the curved guide and the lens make the position of this minimum a function of the particle velocity. As a consequence, the beam density normalisation as described above works well for any given velocity, but unless the effect here is taken into account it does not work when comparing two different beam velocities. In order to do so, plots like Fig. 13 have to be recorded for each velocity of the polar beam, and the ratio between ion signal and REMPI signal needs to be added as an additional factor in the normalisation procedure. Doing this properly means that a large velocity range that covers multiple expansion conditions for both molecular beams can be covered and joined together, based entirely on experimental data, to match perfectly (see Fig. 11b).

It is important to note that these measurements are required to correct for an error that would otherwise be introduced in the normalization, they are not the normalization itself. We measure the amplitude of the REMPI signal for different focusing conditions at one particular relative velocity. Because of this we know that the observed reaction rate coefficient must remain constant, and any change we observe is due to the effect described above that distorts the normalization. These measurements thus provide an experimental scaling factor that needs to be included in order to take the different beam trajectories correctly into account.

An alternative solution would be to measure the beam density directly at the interaction zone. This, however, might interfere with the correct counting of reaction products, and it

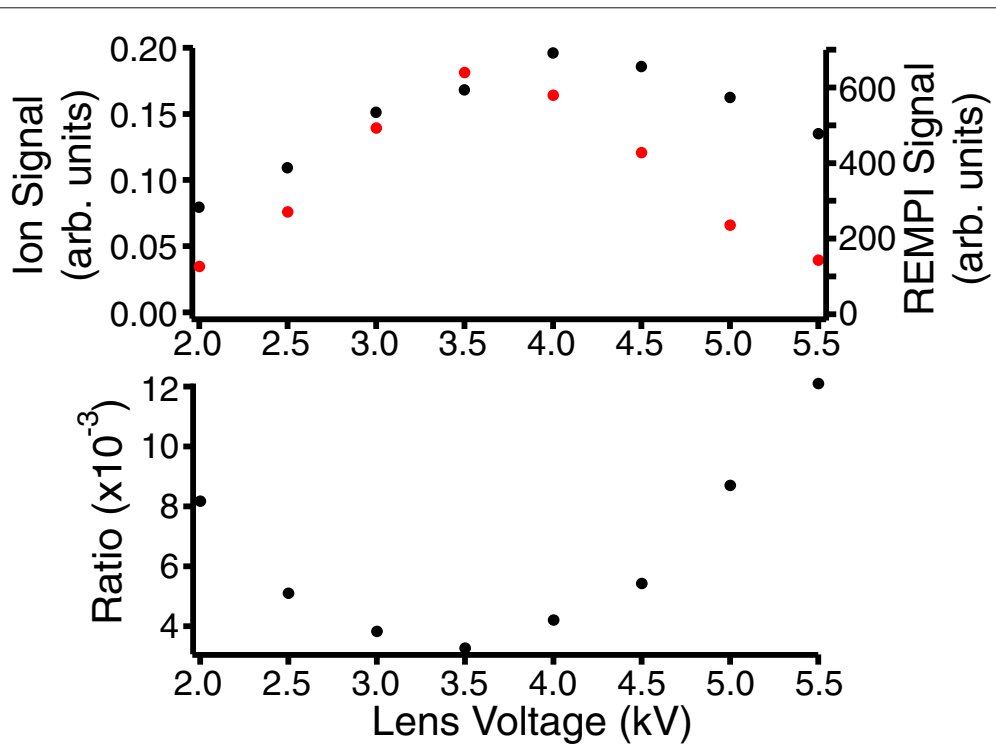

Fig. 13 Experimental characterization of the beam density normalisation for different voltages on the electrostatic lens. Top panel: ion signal (red dots, left scale) and REMPI signal (black dots, right scale) for $\mathrm{NH}_{3}$ molecules being ionized either by Penning ionisation with $\mathrm{Ne}\left({ }^{3} \mathrm{P}_{2}\right)$ or by REMPI, and focused with different lens voltages. Bottom panel: Ratio of the two signals from the first panel as a function of lens voltage 
would be very difficult to measure the density of that component of the molecular packet that was actually probed through the collisions.

\section{Outlook}

Since this review focuses on technical aspects of the merged-beam experiments we also restrict the outlook on possible experimental improvements and developments, rather than on future applications. This section lists a few aspects that will benefit from improvements and where such an improvement can be possible without fundamentally changing the experiment. We discuss these aspects per section of the chamber.

\section{Source}

From our reasoning above and reference [49] it is obvious that short pulses with high speed ratio are desired in order to get the highest-possible resolution. Current supersonic expansions provide pulses as short as $10 \mu$ s and speed ratios exceeding 100 in some cases. Higher beam densities would lead to improved sensitivity and/or reduced acquisition times. Unfortunately, the current conditions achieved with modern supersonic expansions are close to the maximum possible in terms of density and flux. Higher densities would inadvertently lead to clustering in the expansion, in particular when polar and easily polarisable molecules are expanded.

Considerably higher speed ratios can be achieved if the supersonic expansion alone is replaced with a Stark or Zeeman decelerator [1]. These devices provide speed ratios of several 100 without necessarily reducing the number density by much. In the current experiment they would not primarily be used to substantially slow molecular beams, but merely to select a specific portion from the original expansion and bring it to an extremely well-defined velocity. It is, in fact, also of interest to slow them since then the merging can be achieved in a very compact guide, and because the ultimate resolution is improved when using slow beams, also in the merged beam technique [25, 68].

Further enhancement of the resolution can be achieved by installing so-called bunchers at the end of the guide. Such devices manipulate the phase-space distribution of molecules in a way that either the spatial or the velocity spread are minimised. The latter would lead to a direct improvement of the maximum resolution possible in a merged beam experiment.

\section{Detection}

The current, quite simple detection, is limited to detecting either total cross sections when the reaction product is an ion, or state-specific cross sections when REMPI is being used. A considerable improvement will result from replacing the TOF-MS with a velocitymap imaging (VMI) detector to measure differential cross sections, as it has become standard in crossed-beam experiments over the past years [82-85].

For neutral reaction products an additional velocity gating will have to be introduced to discriminate against products formed at earlier times. If this is omitted then products formed far from the detection point may a) have been formed at an ill-defined relative velocity or b) have moved transversely sufficiently far to lead to blurred images. This problem is eliminated by using sources with very high speedratios (see above) or by adding other devices that select very narrow ranges of velocities. 


\section{Towards neutral reaction products}

One big challenge in this technique currently lies in the extension neutral to reaction products. The Penning ionisation studies performed to date benefitted from the simple and efficient detection of ions which means that even low numbers of reaction products can be monitored very efficiently. The detection of neutral reaction products requires an additional step, where the neutral is either ionized by REMPI or excited to be detected by Laser-induced fluorescence (LIF). Both of these processes have a limited total efficiency. Given that the number of reaction products produced at every experimental cycle is low already (single digits in certain cases of the systems studied here), both REMPI and LIF are not realistic. The solution will be to either develop new, ultra sensitive detection methods that enable the counting basically of single molecules, or do substantially increase the densities and thus the observed reaction rates.

\section{Conclusions}

The merged beam technique has successfully been developed for the application to neutral reactions. While it has been applied to ion-neutral reactions already several decades ago, substantial additional developments were necessary to implement the neutral version. Stark deceleration and Zeeman deceleration, both implemented in the past 15 years, have lead to perfection of the tools to guide neutral molecules and bend supersonic beams. The particular application of guiding one beam onto the axis of another one finally permitted to break through the $1 \mathrm{~K}$ barrier in neutral reactions using supersonic expansions.

In the present review we have given a detailed account of the construction and implementation of our merged beam apparatus. The currently only other existing such device is very similar in principle, but differs in many details. For details about that apparatus we refer to articles published by Narevicius et al. $[45,49,50]$. Both these experiments have produced several studies of neutral reactions already, and they point the way for future investigations. At the same time, these first experiments also show what direction future technical improvements must take. We have listed some of the extensions that can be expected to be implemented in the next few years, either as modifications to the EPFLand Weizmann-setups, or as new constructions in other labs. All these improvements will further push our ability to perform detailed studies of gas-phase reactions at low collision energies, ultimately reaching the final goal to study reactions between large, oriented, state-selected neutral molecules at well-defined and potentially very low collision energies with full resolution of state-to-state differential cross sections. Exciting times lay ahead of us indeed.

Competing interests

The authors declare that they have no competing interests.

\section{Acknowledgements}

The design and construction of this experiment were the core work of Dr. Benjamin Bertsche during his time as a PhD student. Most of the experimental results described in this article were collected by Dr. Justin Jankunas, assisted by Kevin R. Reisyan. Their invaluable contribution is greatly appreciated.

This work would not have been possible without the expert support from the mechanical workshop at the institute for chemical sciences and engineering at EPFL. We would most particularly like to thank André Fattet (head of the workshop), Yves Morier, Christophe Clément, and Gil Corbaz for their help. Financial support was provided by the Swiss National Science Foundation (grant number PP0022-119081) and by EPFL. 


\section{References}

1. van de Meerakker SYT, Bethlem HL, Vanhaecke N, Meijer G. Manipulation and control of molecular beams. Chem Rev. 2012;112(9):4828-78.

2. Lemeshko M, Krems RV, Doyle JM, Kais S. Manipulation of molecules with electromagnetic fields. Mol Phys. 2013;111(12-13):1648-82.

3. Jankunas J, Osterwalder A. Cold and Controlled Molecular Beams: Production and Applications. Annu Rev Phys Chem. 2014;66(1):241-62.

4. Krems RV, Friedrich B, Stwalley WC. Cold Molecules: Theory, Experiment, Applications. Boca Raton: CRC Press; 2009.

5. Sims IR, Smith I. Gas-Phase Reactions and Energy Transfer at Very Low Temperatures. Annu Rev Phys Chem. 1995;46:109-37.

6. Smith I. Low Temperatures and Cold Molecules. London: Imperial College Press; 2008.

7. Chandler DW. Cold and ultracold molecules: Spotlight on orbiting resonances. J Chem Phys. 2010;132(11):110901.

8. Hutson JM. Ultracold Chemistry. Science. 2010;327(5967):788-9.

9. Smith I. Laboratory Astrochemistry: Gas-Phase Processes. Annu Rev Astro Astrophys. 2011;49(1):29-66.

10. Brouard M, Parker DH, van de Meerakker SYT. Taming molecular collisions using electric and magnetic fields. Chem Soc Rev. 2014;43(21):7279-94.

11. Stuhl BK, Hummon MT, Ye J. Cold State-Selected Molecular Collisions and Reactions. Annu Rev Phys Chem. 2014;65:501-18.

12. Ospelkaus S, Ni KK, Wang D, de Miranda MHG, Neyenhuis B, Quéméner G, Julienne PS, Bohn JL, Jin DS, Ye J. Quantum-state controlled chemical reactions of ultracold potassium-rubidium molecules. Science. 2010;327(5967):853-7.

13. Knoop S, Ferlaino F, Berninger M, Mark M, Naegerl HC, Grimm R, D'incao JP, Esry BD. Magnetically Controlled Exchange Process in an Ultracold Atom-Dimer Mixture. Phys Rev Lett. 2010;104(5):053201.

14. de Miranda MHG, Chotia A, Neyenhuis B, Wang D, Quemener G, Ospelkaus S, Bohn J, Ye J, Jin D. Controlling the quantum stereodynamics of ultracold bimolecular reactions. Nat Phys. 2011;7(6):502-7.

15. Scoles G. Atomic and Molecular Beam Methods. Oxford: Oxford University Press; 1988

16. Levine R. Molecular Reaction Dynamics. Cambridge: Cambridge University Press; 2009.

17. Brouard M, Vallance C. Tutorials in Molecular Reaction Dynamics. Cambridge: Royal Society of Chemistry; 2011

18. Chefdeville S, Stoecklin T, Bergeat A, Hickson KM, Naulin C, Costes M. Appearance of Low Energy Resonances in CO-Para-H ${ }_{2}$ Inelastic Collisions. Phys Rev Lett. 2012;109(2):23201.

19. Chefdeville S, Kalugina Y, van de Meerakker SYT, Naulin C, Lique F, Costes M. Observation of partial wave resonances in low-energy $\mathrm{O}_{2}-\mathrm{H}_{2}$ inelastic collisions. Science. 2013;341(6150):1094-6.

20. Bergeat A, Onvlee J, Naulin C, van der Avoird A, Costes M. Quantum dynamical resonances in low-energy $\mathrm{CO}(\mathrm{j}=0)+\mathrm{He}$ inelastic collisions. Nat Chem. 2015;7(4):349-53.

21. Sawyer BC, Stuhl BK, Yeo M, Tscherbul TV, Hummon MT, Xia Y, Kłos J, Patterson D, Doyle JM, Ye J. Cold heteromolecular dipolar collisions. Phys Chem Chem Phys. 2011;13(42):19059-66.

22. Trujillo SM, Neynaber RH, Rothe EW. Merging beams, a different approach to collision cross section measurements. Rev Sci Instrum. 1966;37(12):1655.

23. Gentry WR, McClure DJ, Douglass CH. Merged beams at Minnesota. Rev Sci Instrum. 1975;46(4):367-75.

24. Phaneuf RA, Havener CC, Dunn GH, Müller A. Merged-beams experiments in atomic and molecular physics. Rep Prog Phys. 1999;62(7):1143-80.

25. Glenewinkel-Meyer T, Gerlich D. Single and Merged Beam Studies of the Reaction $\mathrm{H}_{2}^{+}(\mathrm{V}=0,1 ; \mathrm{j}=0,4)+\mathrm{H}_{2} \rightarrow \mathrm{H}_{3}^{+}+$ H. Isr J Chem. 1997;37:343

26. Bethlem HL, Berden G, Meijer G. Decelerating Neutral Dipolar Molecules. Phys Rev Lett. 1999;83(8):1558-61.

27. Bethlem HL, Berden G, Crompvoets F, Jongma RT, Van Roij A, Meijer G. Electrostatic trapping of ammonia molecules. Nature. 2000;406:491-4.

28. Bochinski J, Hudson ER, Lewandowski H, Meijer G, Ye J. Phase Space Manipulation of Cold Free Radical OH Molecules. Phys Rev Lett. 2003;91(24):243001.

29. Hudson ER, Bochinski J, Lewandowski H, Sawyer BC, Ye J. Efficient Stark deceleration of cold polar molecules. Eur Phys J D. 2004;31(2):351-8.

30. Lewandowski H, Hudson ER, Bochinski J, Ye J. A pulsed, low-temperature beam of supersonically cooled free radical $\mathrm{OH}$ molecules. Chem Phys Lett. 2004;395:53-7.

31. Vanhaecke N, Meier U, Andrist M, Meier BH, Merkt F. Multistage Zeeman deceleration of hydrogen atoms. Phys Rev A. 2007;75(3):031402.

32. Narevicius E, Parthey C, Libertun AR, Riedel MF, Even U, Raizen MG. Towards magnetic slowing of atoms and molecules. New J Phys. 2007;9(4):96.

33. Meek SA, Conrad H, Meijer G. Trapping Molecules on a Chip. Science. 2009;324(5935):1699-1702.

34. Osterwalder A, Meek SA, Haak H, Meijer G. Deceleration of neutral molecules in macroscopic traveling traps. Phys Rev A. 2010;81(5):051401.

35. Meek SA, Parsons MF, Haak H, Meijer G, Osterwalder A. A traveling wave decelerator for neutral polar molecules. Rev Sci Instrum. 2011;82(9):093108.

36. Lavert-Ofir E, Gersten S, Henson AB, Shani I, David L, Narevicius J, Narevicius E. A moving magnetic trap decelerator: a new source of cold atoms and molecules. New J Phys. 2011;13(10):103030.

37. Dulitz K, Tauschinsky A, Softley TP. Zeeman deceleration of electron-impact-excited metastable helium atoms. New J Phys. 2015;17(3):035005.

38. Dulitz K, Motsch M, Vanhaecke N, Softley TP. Getting a grip on the transverse motion in a Zeeman decelerator. J Chem Phys. 2014;140(10):104201.

39. Rangwala SA, Junglen T, Rieger T, Pinkse PWH, Rempe G. Continuous source of translationally cold dipolar molecules. Phys Rev A. 2003;67(4):043406.

40. Junglen T, Rieger T, Rangwala SA, Pinkse PWH, Rempe G. Slow ammonia molecules in an electrostatic quadrupole guide. Eur Phys J D. 2004;31(2):365-73. 
41. Rieger T, Junglen $T$, Rangwala SA, Pinkse PWH, Rempe G. Continuous loading of an electrostatic trap for polar molecules. Phys Rev Lett. 2005;95(17):173002.

42. Rieger T, Junglen T, Rangwala SA, Rempe G, Pinkse PWH, Bulthuis J. Water vapor at a translational temperature of 1 K. Phys Rev A. 2006;73(6):061402.

43. Bertsche B, Osterwalder A. State-selective detection of velocity-filtered $N_{3}$ molecules. Phys Rev A. 2010;82(3): 033418.

44. Bertsche B, Osterwalder A. Dynamics of individual rotational states in an electrostatic guide for neutral molecules. Phys Chem Chem Phys. 2011;13(42):18954.

45. Henson AB, Gersten S, Shagam Y, Narevicius J, Narevicius E. Observation of Resonances in Penning lonization Reactions at Sub-Kelvin Temperatures in Merged Beams. Science. 2012;338:234-8.

46. Bertsche B. Low Energy Collisions in Merged Neutral Molecular Beams. PhD thesis, EPFL, Lausanne. 2014. https://infoscience.epfl.ch/record/199466? In=fr.

47. Jankunas J, Bertsche B, Jachymski K, Hapka M, Osterwalder A. Dynamics of gas phase $\mathrm{Ne}^{*}+\mathrm{NH}_{3}$ and $\mathrm{Ne}^{*}+\mathrm{ND}_{3}$ Penning ionisation at low temperatures. J Chem Phys. 2014;140(24):244302.

48. Wei Q, Lyuksyutov I, Herschbach D. Merged-beams for slow molecular collision experiments. J Chem Phys. 2012;137(5):054202.

49. Shagam Y, Narevicius E. Sub-Kelvin Collision Temperatures in Merged Neutral Beams by Correlation in Phase-Space. J Phys Chem C. 2013;117(43):22454-61.

50. Lavert-Ofir E, Shagam Y, Henson AB, Gersten S, Kłos J, Zuchowski P, Narevicius J, Narevicius E. Observation of the isotope effect in sub-kelvin reactions. Nat Chem. 2014;6(4):332-5.

51. Jankunas J, Jachymski K, Hapka M, Osterwalder A. Observation of orbiting resonances in He(3S1) + NH3 Penning ionization. J Chem Phys. 2015;142(16):164305.

52. Jankunas J, Bertsche B, Osterwalder A. Study of the $\mathrm{Ne}\left({ }^{3} \mathrm{P}_{2}\right)+\mathrm{CH}_{3}$ F Electron-Transfer Reaction below $1 \mathrm{~K}$. J Phys Chem A. 2014;118(22):3875-9.

53. Bertsche B, Jankunas J, Osterwalder A. Low-temperature Collisions between Neutral Molecules in Merged Molecular Beams. Chimia. 2014;68(4):256-9.

54. Allmendinger P, Deiglmayr J, Agner JA, Schmutz H, Merkt F. Surface-electrode decelerator and deflector for Rydberg atoms and molecules. Phys Rev A. 2014;90(4):043403.

55. Bethlem HL. private communication.

56. Crompvoets F, Bethlem HL, Meijer G. A Storage Ring for Neutral Molecules. Adv Atom Mol Opt Phys. 2005;52:209.

57. van de Meerakker SYT, Bethlem HL, Meijer G. Taming molecular beams. Nat Phys. 2008;4(8):595-602.

58. Carr L, DeMille D, Krems RV, Ye J. Cold and ultracold molecules: science, technology and applications. New J Phys. 2009;11:055049.

59. Parker D, Bernstein RB. Oriented molecule beams via the electrostatic hexapole: Preparation, characterization, and reactive scattering. Annu Rev Phys Chem. 1989;40(1):561-95.

60. Niehaus A. Spontaneous lonization in Slow Collisions. Adv Chem Phys. 1981;45:399.

61. Siska PE. Molecular-beam studies of Penning ionization. Rev Mod Phys. 1993;65(2):337-412.

62. Lu HI, Rasmussen J, Wright MJ, Patterson D, Doyle JM. A cold and slow molecular beam. Phys Chem Chem Phys. 2011;13(42):18986-90.

63. Patterson D, Rasmussen J, Doyle JM. Intense atomic and molecular beams via neon buffer-gas cooling. New J Phys. 2009;11:055018.

64. Patterson D, Doyle JM. Bright, guided molecular beam with hydrodynamic enhancement. J Chem Phys. 2007;126(15):154307.

65. Steer EW, Twyman KS, Heazlewood BR, Softley TP. Accurate determination of the relative concentrations of ammonia isotopologues in a cold, electrostatically guided molecular beam. Mol Phys. 2015;113(12):1465-71.

66. Twyman KS, Bell M, Heazlewood BR, Softley TP. Production of cold beams of ND3 with variable rotational state distributions by electrostatic extraction of He and Ne buffer-gas-cooled beams. J Chem Phys. 2014;141 (2):024308.

67. Shagam Y, Narevicius E. Sub-Kelvin Collision Temperatures in Merged Neutral Beams by Correlation in Phase-Space. J Phys Chem C. 2013;117:22454-61.

68. Gerlich D. Kinematic averaging effects in thermal and low energy ion-molecule collisions: Influence on product ion kinetic energy distributions. J Chem Phys. 1989;90(1):127-39.

69. Jankunas J, Reisyan KS, Osterwalder A. Preparation of State Purified Beams of $\mathrm{He}, \mathrm{Ne}, \mathrm{C}, \mathrm{N}$, and O Atoms. J Chem Phys. 2015;142:10311.

70. Rieger T, Rangwala SA, Pinkse PWH, Rempe G. Two-dimensional trapping of dipolar molecules in time-varying electric fields. Phys Rev Lett. 2004;92(22):223001.

71. Putzke S, Filsinger F, Haak H, Küpper J, Meijer G. Rotational-state-specific guiding of large molecules. Phys Chem Chem Phys. 2011;13(42):18962.

72. Filsinger F, Putzke $\mathrm{S}$, Haak H, Meijer $\mathrm{G}$, Küpper J. Optimizing the resolution of the alternating-gradient $\mathrm{m} / \mu$ selector. Phys Rev A. 2010;82(5):052513.

73. Zieger P, van de Meerakker SYT, Heiner CE, Bethlem HL, Van Roij A, Meijer G. Multiple Packets of Neutral Molecules Revolving for over a Mile. Phys Rev Lett. 2010;105(17):173001.

74. Luria K, Lavie N, Even U. Dielectric barrier discharge source for supersonic beams. Rev Sci Instrum. 2009;80(10): 104102.

75. Yan B, Claus PFH, van Oorschot BGM, Gerritsen L, Eppink ATJB, van de Meerakker SYT, Parker DH. A new high intensity and short-pulse molecular beam valve. Rev Sci Instrum. 2013;84(2):023102.

76. COMSOL Multiphysics: different versions. Comsol AB. 2009-2015.

77. Jankunas J, Reisyan KS, Osterwalder A. Preparation of state purified beams of $\mathrm{He}, \mathrm{Ne}, \mathrm{C}, \mathrm{N}$, and $\mathrm{O}$ atoms. J Chem Phys. 2015;142(10):104311.

78. Motsch M, Sommer C, Zeppenfeld M, van Buuren LD, Pinkse PWH, Rempe G. Collisional effects in the formation of cold guided beams of polar molecules. New J Phys. 2009;11:055030. 
79. Motsch M, van Buuren LD, Sommer C, Zeppenfeld M, Rempe G, Pinkse PWH. Cold guided beams of water isotopologs. Phys Rev A. 2009;79(1):013405.

80. Motsch M, Scheibner KF, Zeppenfeld M, Schmitt M, Meerts LW, Pinkse PWH, Rempe G. Spectroscopy of the $\tilde{A}^{1} A_{2} \leftarrow \tilde{X}^{1} A_{1}$ transition of formaldehyde in the $30140-30790 \mathrm{~cm}^{-1}$ range: The $2{ }_{0}^{1} 4_{0}^{3}$ and $2_{0}^{2} 4_{0}^{1}$ rovibrational bands. J Mol Spect. 2008;252(1):25-30

81. Heiner CE, Meijer G, Bethlem HL. Motional resonances in a molecular synchrotron. Phys Rev A. 2008;78(3):030702.

82. Eppink A, Parker DH. Velocity map imaging of ions and electrons using electrostatic lenses: Application in photoelectron and photofragment ion imaging of molecular oxygen. Rev Sci Instrum. 1997;68:3477.

83. Li W, Huang C, Patel M, Wilson D, Suits AG. State-resolved reactive scattering by slice imaging: A new view of the $\mathrm{Cl}+\mathrm{C}_{2} \mathrm{H}_{6}$ reaction. J Chem Phys. 2006;124(1):011102

84. Zhang W, Kawamata H, Liu K. CH Stretching Excitation in the Early Barrier F $+\mathrm{CHD}_{3}$ Reaction Inhibits $\mathrm{CH}$ Bond Cleavage. Science. 2009;325(5938):303-306.

85. von Zastrow A, Onvlee J, Vogels SN, Groenenboom GC, van der Avoird A, van de Meerakker SYT. State-resolved diffraction oscillations imaged for inelastic collisions of NO radicals with He, Ne and Ar. Nat Chem. 2014;6(3):216-21.

\section{Submit your manuscript to a SpringerOpen ${ }^{\odot}$} journal and benefit from:

- Convenient online submission

- Rigorous peer review

- Immediate publication on acceptance

- Open access: articles freely available online

- High visibility within the field

- Retaining the copyright to your article

Submit your next manuscript at $\gg$ springeropen.com 